\title{
Superconformal flavor simplified
}

\section{David Poland and David Simmons-Duffin}

Jefferson Physical Laboratory, Harvard University, Cambridge, Massachusetts 02138, U.S.A.

E-mail: dpoland@physics.harvard.edu, davidsd@physics.harvard.edu

ABSTRACT: A simple explanation of the flavor hierarchies can arise if matter fields interact with a conformal sector and different generations have different anomalous dimensions under the CFT. However, in the original study by Nelson and Strassler many supersymmetric models of this type were considered to be 'incalculable' because the $R$-charges were not sufficiently constrained by the superpotential. We point out that nearly all such models are calculable with the use of $a$-maximization. Utilizing this, we construct the simplest vector-like flavor models and discuss their viability. A significant constraint on these models comes from requiring that the visible gauge couplings remain perturbative throughout the conformal window needed to generate the hierarchies. However, we find that there is a small class of simple flavor models that can evade this bound.

KeYwords: Supersymmetric gauge theory, Quark Masses and SM Parameters, Renormalization Group

ArXiv ePrint: 0910.4585 


\section{Contents}

1 Introduction 1

2 Overview 3

2.1 Review of flavor physics 3

2.2 Superconformal flavor models 5

2.2.1 Toy model 6

2.3 Review of $a$-maximization $\quad 7$

2.3.1 $a$-maximization in the toy model 9

3 Vector-like models $\quad 10$

$\begin{array}{lll}3.1 & \mathbf{1 0}+\overline{\mathbf{5}}+\mathbf{1} \text { model } & 13\end{array}$

$\begin{array}{lll}3.2 & \mathrm{SU}(\mathrm{N}) \text { with an adjoint } & 15\end{array}$

$\begin{array}{ll}\text { 3.2.1 Deformation by } \operatorname{Tr}\left[A^{3}\right] & 17\end{array}$

$\begin{array}{lll}3.3 & \mathrm{Sp}(2 \mathrm{~N}) \text { with an Anti-symmetric Tensor } & 18\end{array}$

$\begin{array}{lll}\text { 3.3.1 Deformation by } \operatorname{Tr}\left[A^{k+1}\right] & 19\end{array}$

$\begin{array}{lll}3.3 .2 & \text { Dual description } & 20\end{array}$

$\begin{array}{ll}\text { 3.3.3 Coupling to } \overline{\mathbf{5}} \text { 's } & 21\end{array}$

4 A previously 'incalculable' model 22

5 Conclusions $\quad 24$

A Sketch of a proof of $a$-maximization $\quad 26$

A.1 Proof of eq. (A.2) 26

A.1.1 Determining $\left\langle T_{\mu} T_{\nu} J_{I}\right\rangle$ from superconformal invariance 27

$\begin{array}{lll}\text { A.2 Proof of eq. (A.3) } & 29\end{array}$

$\begin{array}{ll}\text { B CFT exit and dual descriptions } & 30\end{array}$

C Models with two 3-field or 4-field $T_{i}$ couplings

$\begin{array}{lll}\text { C.1 Models without 10's } & 35\end{array}$

$\begin{array}{lll}\text { C.2 Models with 10's } & 36\end{array}$

\section{Introduction}

While the Standard Model is highly successful as an effective field theory in describing nature, one of its big mysteries is the observed pattern of quark and lepton masses and mixing angles. This puzzle remains even after new physics such as supersymmetry or strong dynamics is introduced in order to solve the hierarchy problem. In fact, there is 
quite often a tension between solutions to the hierarchy problem and a successful picture of flavor physics, leading to many models that, while quite interesting, perhaps seem more contrived than what nature should allow.

A simple explanation of the structure of flavor physics can come about if the Yukawa interactions are anarchical in the UV but each field comes with an additional suppression factor in the IR effective theory [1-4]. These suppression factors can arise in 4D theories if each field carries a different anomalous dimension under strong conformal dynamics $[5,6]$, or in warped 5D theories [7] if each field has a different exponential profile as determined by its bulk mass $[8,9]$. In fact, these two pictures can be related via the AdS/CFT correspondence [10-14]. The bulk masses in 5D theories are usually taken to be free parameters, leading to a successful explanation of flavor physics, but leaving open the question of whether there exists a dual 4D CFT containing operators with the corresponding anomalous dimensions.

On the other hand, one can attempt to find concrete 4D theories in which the anomalous dimensions can be determined as output, rather than input. Some examples of this in a supersymmetric context were given in [6]. However, these models were necessarily somewhat complicated because, unless there were a large number of marginal interactions in the superpotential, there was no general method available to determine the anomalous dimensions (or equivalently the $R$-charges) of every field.

Here we point out that $a$-maximization [15] is precisely the needed method, because it allows one to determine the correct superconformal $\mathrm{U}(1)_{\mathrm{R}}$-symmetry even when there are only a small number of additional constraints. We will demonstrate that this allows one to construct very simple calculable models of flavor physics, as well as reanalyze the 'incalculable' models presented in [6].

However, since these models inevitably introduce many new multiplets charged under $\mathrm{SU}(3) \times \mathrm{SU}(2) \times \mathrm{U}(1)$, this flavor physics must occur at a high scale in order for the visible gauge couplings to remain perturbative. In addition, many of these models introduce couplings that violate baryon and lepton number, potentially inducing problematic protondecay operators. A simple way to avoid both of these constraints is to assume that the conformal dynamics occurs above the GUT scale, and thus we focus on on finding the simplest vector-like flavor models in the context of $\mathrm{SU}(5)_{\text {GUT }}$ unification. We will find that, even in this case, a significant constraint comes from requiring that the $\mathrm{SU}(5)_{\text {GUT }}$ gauge coupling remain perturbative throughout the entire conformal window. However, we will demonstrate that a small class of models can also satisfy this constraint while explaining the observed flavor hierarchies.

The outline of this work is as follows. In section 2 we will give brief reviews of flavor physics and $a$-maximization. In section 3 we study simple concrete flavor models with vector-like matter content. In section 4 we analyze one of the previously 'incalculable' models presented by Nelson and Strassler. We conclude in section 5 . 


\section{Overview}

\subsection{Review of flavor physics}

We will start with a brief review of how the flavor hierarchies appear in supersymmetric extensions of the Standard Model. The Yukawa couplings of the matter fields $Q_{i}, U_{i}, D_{i}, L_{i}, E_{i}$ to the Higgs fields $H_{u}, H_{d}$ arise from the superpotential operators

$$
W=y_{u}^{i j} Q_{i} U_{j} H_{u}+y_{d}^{i j} Q_{i} D_{j} H_{d}+y_{l}^{i j} L_{i} E_{j} H_{d}\left(+y_{N}^{i j} L_{i} N_{j} H_{u}+M_{N}^{i j} N_{i} N_{j}\right),
$$

where the matrices $y_{a}^{i j}$ possess a hierarchical structure in the basis where all of the matter fields have a canonically normalized Kähler potential. (Here we have included the possibility of generating neutrino masses via the seesaw mechanism by integrating out massive right-handed neutrinos $N_{i}$.)

There are two fundamentally different ways that this hierarchical structure could arise from an underlying flavor physics model. The first is that the model genuinely generates a hierarchical structure in the superpotential while not significantly affecting the Kähler potential. This could happen, e.g., in models in which the couplings of the lighter generations ultimately arise from higher dimensional operators in the superpotential [2-4]. In this case one would need to know the details of the model in order to determine if there are any predicted relations between the masses and mixing angles.

The second possibility is that the superpotential contains no hierarchical structure ("flavor anarchy"), but the underlying flavor physics model generates a hierarchy in the wave-function factors

$$
\mathcal{L}=\int d^{4} \theta \sum_{i} Z_{i} \Phi_{i}^{\dagger} \Phi_{i}
$$

with, e.g., $Z_{Q_{1}} \gg Z_{Q_{2}} \gg Z_{Q_{3}}$. Taking $\epsilon_{i} \equiv Z_{i}^{-1 / 2}$, then up to $O(1)$ coefficients one obtains the generic mass structure

$$
\begin{aligned}
\left(m_{t}, m_{c}, m_{u}\right) & \approx\left\langle H_{u}\right\rangle\left(\epsilon_{Q_{3}} \epsilon_{U_{3}} \epsilon_{H_{u}}, \epsilon_{Q_{2}} \epsilon_{U_{2}} \epsilon_{H_{u}}, \epsilon_{Q_{1}} \epsilon_{U_{1}} \epsilon_{H_{u}}\right) \\
\left(m_{b}, m_{s}, m_{d}\right) & \approx\left\langle H_{d}\right\rangle\left(\epsilon_{Q_{3}} \epsilon_{D_{3}} \epsilon_{H_{d}}, \epsilon_{Q_{2}} \epsilon_{D_{2}} \epsilon_{H_{d}}, \epsilon_{Q_{1}} \epsilon_{D_{1}} \epsilon_{H_{d}}\right) \\
\left(m_{\tau}, m_{\mu}, m_{e}\right) & \approx\left\langle H_{d}\right\rangle\left(\epsilon_{L_{3}} \epsilon_{E_{3}} \epsilon_{H_{d}}, \epsilon_{L_{2}} \epsilon_{E_{2}} \epsilon_{H_{d}}, \epsilon_{L_{1}} \epsilon_{E_{1}} \epsilon_{H_{d}}\right) \\
\left(m_{\nu_{\tau}}, m_{\nu_{\mu}}, m_{\nu_{e}}\right) & \approx \frac{\left\langle H_{u}\right\rangle^{2}}{M_{N}}\left(\epsilon_{L_{3}}^{2} \epsilon_{H_{u}}^{2}, \epsilon_{L_{2}}^{2} \epsilon_{H_{u}}^{2}, \epsilon_{L_{1}}^{2} \epsilon_{H_{u}}^{2}\right),
\end{aligned}
$$

as well as the mixing angles

$$
\left|V_{\mathrm{CKM}}\right| \approx\left(\begin{array}{ccc}
1 & \epsilon_{Q_{1}} / \epsilon_{Q_{2}} & \epsilon_{Q_{1}} / \epsilon_{Q_{3}} \\
\epsilon_{Q_{1}} / \epsilon_{Q_{2}} & 1 & \epsilon_{Q_{2}} / \epsilon_{Q_{3}} \\
\epsilon_{Q_{1}} / \epsilon_{Q_{3}} & \epsilon_{Q_{2}} / \epsilon_{Q_{3}} & 1
\end{array}\right),\left|V_{\mathrm{MNS}}\right| \approx\left(\begin{array}{ccc}
1 & \epsilon_{L_{1}} / \epsilon_{L_{2}} & \epsilon_{L_{1}} / \epsilon_{L_{3}} \\
\epsilon_{L_{1}} / \epsilon_{L_{2}} & 1 & \epsilon_{L_{2}} / \epsilon_{L_{3}} \\
\epsilon_{L_{1}} / \epsilon_{L_{3}} & \epsilon_{L_{2}} / \epsilon_{L_{3}} & 1
\end{array}\right)
$$

This generic structure can well accommodate the present data. For example, the observed mixing angles (taken from [16] and [17]) are given by

$$
\left|V_{\mathrm{CKM}}\right| \simeq\left(\begin{array}{ccc}
0.97 & 0.23 & 0.004 \\
0.23 & 0.97 & 0.04 \\
0.009 & 0.04 & 0.99
\end{array}\right),\left|V_{\mathrm{MNS}}\right| \simeq\left(\begin{array}{ccc}
0.77-0.86 & 0.50-0.63 & 0.00-0.22 \\
0.22-0.56 & 0.44-0.73 & 0.57-0.80 \\
0.21-0.55 & 0.40-0.71 & 0.59-0.82
\end{array}\right)
$$


It is particularly interesting to note that this generic structure predicts that these matrices are symmetric up to $O(1)$ effects, and hence prefers a larger value of $\theta_{13}$ in the neutrino sector.

In this paper, we'll focus primarily on supersymmetric SU(5) GUT models, with three SM generations $T_{i} \in \mathbf{1 0}$ and $\bar{F}_{i} \in \overline{\mathbf{5}}$, and Higgs fields $H \in \mathbf{5}, \bar{H} \in \overline{\mathbf{5}}$. When SU(5) GUT relations are satisfied at the unification scale $M_{\mathrm{GUT}} \sim 10^{16} \mathrm{GeV}$, one has the relations

$$
\epsilon_{Q_{i}}=\epsilon_{U_{i}}=\epsilon_{E_{i}} \equiv \epsilon_{T_{i}} \quad \text { and } \quad \epsilon_{D_{i}}=\epsilon_{L_{i}} \equiv \epsilon_{\bar{F}_{i}} .
$$

Considering the GUT values of the up-type quark masses then yields the estimates (extracted from ref. [18] at $\tan \beta \sim 30$ )

$$
\epsilon_{T_{i}} \sqrt{\epsilon_{H}} \approx(.001-.002, .03-.04, .7-.8)
$$

Note however that a somewhat larger value of $\epsilon_{T_{1}}$ (by a factor of $\sim 4-5$ ) is preferred by the Cabibbo angle in eq. (2.5).

Similarly, considering the GUT values of the down-type quark masses yields the estimates

$$
\epsilon_{\bar{F}_{i}} \epsilon_{\bar{H}} \approx \tan \beta \times(.002-.01, .001-.007, .006-.02),
$$

while considering the lepton masses gives

$$
\epsilon_{\bar{F}_{i}} \epsilon_{\bar{H}} \approx \tan \beta \times(.001-.002, .006-.01, .01-.03) .
$$

Here we have tried to illustrate the potentially large uncertainties arising from SUSY threshold corrections, which can be important at larger values of $\tan \beta$. In most cases, an $O(1)$ violation of the GUT relations in the first and second generations will be required to be consistent with low-energy data. Nevertheless, it is interesting that the estimates of eqs. (2.8) and (2.9) are not hierarchically different from one another. In particular, it is still entirely possible that the suppression factors arising from the Kähler potential respect $\mathrm{SU}(5)_{\text {GUT }}$ relations but the anarchical superpotential couplings do not. Moreover, it may still be possible that $\mathrm{SU}(5)_{\text {GUT }}$ relations are allowed in the presence of large SUSY threshold corrections [18-25]. In the present work, we will not present a completely realistic GUT model or discuss the origin of SUSY-breaking parameters, and so will for the most part attempt to remain agnostic about how $O(1)$ violations of these relations may arise.

Perhaps the minimal potentially realistic structure consistent with these estimates is a '10-centered' model, or a model which generates suppressions in $\epsilon_{T_{1}}$ and $\epsilon_{T_{2}}$. In the simplest version, the smallness of the down-type masses must be generated through a combination of large $\tan \beta$ and somewhat small $O(1)$ factors. Smaller $\tan \beta$ is possible, however, if the model additionally generates suppressed values of $\epsilon_{\bar{H}}$ or $\epsilon_{\bar{F}_{i}}$. In this case, a somewhat small value of $\epsilon_{\bar{F}_{1}}$ relative to $\epsilon_{\bar{F}_{2,3}}$ may also be preferred to explain the smallness of $m_{e} / m_{\mu}$. However, since this may not be necessary to explain the ratio of $m_{d} / m_{s}$, this will depend on the details of how GUT-breaking effects are introduced.

While somewhat tangential to the present work, it is interesting to note that the above approach to flavor physics can have important implications for the SUSY flavor problem. If 
the flavor hierarchies simply arise as small numbers in the superpotential (as is commonly assumed), one might worry that generic $M_{\mathrm{pl}}$-suppressed SUSY-breaking operators in the Kähler potential such as $X^{\dagger} X \Phi_{i}^{\dagger} \Phi_{j} / M_{\mathrm{pl}}^{2}$ will lead to large flavor violation in the soft masses if they are the dominant contribution. In this case one is strongly motivated to think about alternative mediation mechanisms such as gauge mediation [26-31]. On the other hand, if the flavor hierarchies arise from the wave-function factors $Z_{i}$, then supersymmetry-breaking operators will also be suppressed by factors of $\epsilon_{i}$ upon canonically normalizing the fields, potentially leading to a simple solution of the flavor problem $[6,32-36]$. Note that in the present scenario these operators can also be further suppressed (or enhanced) because in superconformal theories the anomalous dimension of $\Phi_{i}^{\dagger} \Phi_{j}$ need not be simply related to the anomalous dimension of $\Phi_{i}$.

\subsection{Superconformal flavor models}

The models we will consider in this paper are based on an elegant solution for producing hierarchical suppression factors using strong dynamics, which was first proposed in a supersymmetric context by Nelson and Strassler in [6]. They considered extending the MSSM to include a strongly coupled sector with gauge group $G$. Standard Model fields $\Phi_{i}$ are singlets under $G$, but can develop large anomalous dimensions $\gamma_{i}$ via superpotential couplings to operators in the new sector.

These anomalous dimensions exponentiate into large wave-function factors in the IR, leading to suppression factors

$$
\frac{d \log Z_{i}}{d \log \mu}=-\gamma_{i} \quad \Longrightarrow \quad \epsilon_{\Phi_{i}}=\exp \left(-\frac{1}{2} \int_{\log \Lambda_{c}}^{\log \Lambda} \gamma_{i} d \log \mu\right)
$$

where $\Lambda$ is the UV scale at which the Yukawas are anarchical, and $\Lambda_{c}$ is the scale at which the exotic sector decouples. The anomalous dimensions $\gamma_{i}$ are typically $O(1)$. So to satisfactorily reproduce the SM Yukawa hierarchies we need the strong dynamics to persist over a large range of scales $\Lambda_{c}<\mu<\Lambda$. Following [6], we consider the case where the strong sector gauge and superpotential couplings flow from the UV to an approximate conformal fixed point at $\mu=\Lambda$ and exit the approximately conformal regime at $\mu=\Lambda_{c}$, for instance due to $\Lambda_{c}$-scale masses for the exotic matter charged under $G$.

In the range $\mu \in\left[\Lambda_{c}, \Lambda\right]$, we can compute the anomalous dimensions $\gamma_{i}$ at leading order by ignoring the SM gauge and Yukawa couplings and approximating our theory as a supersymmetric conformal field theory (SCFT) with strongly-coupled gauge group $G$ and an $\mathrm{SU}(5)$ global flavor symmetry. In this approximation, the $\gamma_{i}$ are constant, and eq. (2.10) can be integrated trivially to give

$$
\epsilon_{\Phi_{i}}=\left(\frac{\Lambda_{c}}{\Lambda}\right)^{\frac{1}{2} \gamma_{i}} \quad \text { for each } \Phi_{i}=T_{i}, \bar{F}_{i}, H, \bar{H}
$$

Conformal or near-conformal dynamics is often easiest to understand in a 'physical' basis where we keep the fields canonically normalized $\sqrt{Z_{i}} \Phi_{i} \rightarrow \Phi_{i}$ at each scale $\mu$, rather 


\begin{tabular}{|c|c|c|}
\hline & $\mathrm{SU}(5)_{\mathrm{GUT}}$ & $\mathrm{SU}(\mathrm{N})$ \\
\hline$X+S$ & $10+1$ & $\square$ \\
\hline $\bar{X}+\bar{S}$ & $\overline{\mathbf{1 0}}+1$ & $\bar{\square}$ \\
\hline
\end{tabular}

Table 1. Matter content of a toy model with one light generation.

than a 'holomorphic' basis where the superpotential is not renormalized. ${ }^{1}$ We will use the physical basis for the rest of this paper, so let us take a moment to recover eq. (2.11) using it. In the physical basis, superpotential couplings run according to the anomalous dimensions of the associated operators, while kinetic terms in the Kähler potential are RG invariant. For instance, the Standard Model Yukawa couplings $y_{u}^{i j}$ and $y_{d}^{i j}$ run according to

$$
\begin{aligned}
& \frac{d y_{u}^{i j}}{d \log \mu}=\beta_{y_{u}^{i j}}=\frac{1}{2}\left(\gamma_{T_{i}}+\gamma_{T_{j}}+\gamma_{H}\right) y_{u}^{i j}, \\
& \frac{d y_{d}^{i j}}{d \log \mu}=\beta_{y_{d}^{i j}}=\frac{1}{2}\left(\gamma_{T_{i}}+\gamma_{\bar{F}_{i}}+\gamma_{\bar{H}}\right) y_{d}^{i j} .
\end{aligned}
$$

In the CFT approximation where $\gamma_{i}$ are constant, these integrate to

$$
\begin{aligned}
& y_{u}^{i j}\left(\Lambda_{c}\right)=\left(\frac{\Lambda_{c}}{\Lambda}\right)^{\frac{1}{2}\left(\gamma_{T_{i}}+\gamma_{T_{j}}+\gamma_{H}\right)} y_{u}^{i j}(\Lambda), \\
& y_{d}^{i j}\left(\Lambda_{c}\right)=\left(\frac{\Lambda_{c}}{\Lambda}\right)^{\frac{1}{2}\left(\gamma_{T_{i}}+\gamma_{\bar{F}_{j}}+\gamma_{\bar{H}}\right)} y_{d}^{i j}(\Lambda),
\end{aligned}
$$

which of course agrees with the suppression factors eq. (2.11).

\subsubsection{Toy model}

A particularly attractive feature of superconformal flavor models is that different SM generations can be singled out dynamically by the CFT. Indeed, even with flavor anarchy in the UV, the basic representation theory of the exotic sector can naturally lead to a hierarchy. As an example, let us consider a toy strong sector with $G=\mathrm{SU}(\mathrm{N})$ and matter content given in table 1.

Before coupling to the MSSM (and treating SU(5) GUT as a spectator), this is simply $\mathrm{SU}(\mathrm{N})$ supersymmetric QCD with $N_{f}=11$, which flows to an interacting conformal fixed point when $\frac{3}{2} N<11<3 N$ [38], or equivalently $4 \leq N \leq 7$. However, in addition to the usual SUSY QCD fixed-point, we we can reach other non-trivial fixed points by deforming the theory with relevant operators. For example, we can add a relevant operator $h(\mu) \mu^{3-d} \mathcal{O}$ to the superpotential at a UV scale $\mu$, where $d$ is the classical dimension of $\mathcal{O}$ and we have normalized $h(\mu)$ to be dimensionless. One generally then expects the (initially small) coupling $h(\mu)$ to grow towards the IR, until it approaches a zero of

$$
\beta_{h}=(\operatorname{dim} \mathcal{O}-3) h=\left(d+\frac{\gamma_{\mathcal{O}}}{2}-3\right) h
$$

\footnotetext{
${ }^{1}$ For instance, in a holomorphic basis, gauge couplings run at one loop, while kinetic terms run according to eq. (2.10). At a conformal fixed point, both of these effects are nonzero, but the rescaling anomaly [37] from passing to the physical basis exactly cancels the one-loop gauge-coupling running. It's simpler to stay in the physical basis where neither the kinetic terms nor the gauge couplings run at a conformal fixed-point.
} 
That is, $h(\mu)$ will grow (and other couplings might also change) until the operator $\mu^{3-d} \mathcal{O}$ becomes approximately marginal $(\operatorname{dim} \mathcal{O} \simeq 3)$ and we've reached the vicinity of a new conformal fixed point, with $h(\mu) \simeq h_{*}$.

Our toy exotic sector admits exactly one relevant gauge-invariant coupling to the MSSM,

$$
W_{\text {int }}=h T_{1} \bar{X} S
$$

Note that while there may be flavor anarchy in the UV, only one linear combination of the $T_{i}$ can participate in a relevant coupling to the exotic sector, and we have simply defined that linear combination to be $T_{1}$ without loss of generality. Now suppose our theory flows to a fixed point where the operator $T_{1} \bar{X} S$ becomes marginal. In the next section, we will show that $T_{1}$ has a positive anomalous dimension at this fixed point and thus develops a suppression factor through RG running. The other two generations $T_{2}, T_{3}$ don't couple to the exotic sector, so they have vanishing anomalous dimensions in the SCFT approximation and don't develop suppression factors. Thus this toy model has exactly one light generation.

More formally, the flavor structure is as follows: given an $\mathrm{SU}(3)_{\mathrm{F}}$ flavor symmetry among the $T_{i}$ in the UV (broken only by $O(1)$ Yukawas), we can think of the coupling $h$ as a spurion in the $\overline{\mathbf{3}}$ of $\mathrm{SU}(3)_{\mathrm{F}}$. Picking a direction for $h$ breaks $\mathrm{SU}(3)_{\mathrm{F}} \rightarrow \mathrm{SU}(2)$, singling out a generation. This breaking is $O(1)$ at the scale $\Lambda$, but gets exponentiated by conformal running. This is analogous to warped flavor models, where bulk masses provide an $O(1)$ source of flavor breaking in addition to the Yukawas, but they get exponentiated in the AdS wave-function profiles. However, as noted in the introduction, bulk masses are typically inputs of warped flavor models, whereas anomalous dimensions (and the resulting Yukawa hierarchies) are outputs of the models we consider here.

To make quantitative predictions for flavor physics, we'd like to actually determine the anomalous dimensions of the SM fields that couple to the strong sector. When the scenario of [6] was first proposed, the problem of finding scaling dimensions of chiral operators in an SCFT was unsolved in general, and a number of candidate superconformal flavor models appeared to be 'incalculable'. However since then, Intriligator and Wecht [15] found a simple and widely applicable solution called $a$-maximization, which we'll now review.

\subsection{Review of $a$-maximization}

The $\mathcal{N}=1$ superconformal symmetry group in 4 dimensions is $\mathrm{SU}(2,2 \mid 1)$, which has $\mathrm{SO}(2,4) \times \mathrm{U}(1)_{\mathrm{R}}$ as its bosonic subgroup. Thus, every SCFT necessarily has a distinguished non-anomalous $\mathrm{U}(1)_{\mathrm{R}}$ charge related by supersymmetry to other generators of the conformal group. In particular, the scaling dimension of a chiral operator $\mathcal{O}$ is determined by its $R$-charge, $\operatorname{dim}(\mathcal{O})=\frac{3}{2} R(\mathcal{O})$.

Suppose a SCFT has a U(1) symmetry $R_{0}$ under which the SUSY generators $Q_{\alpha}$ have charge -1 , and a collection of $\mathrm{U}(1)$ "flavor" symmetries $F_{I}$ under which the $Q_{\alpha}$ are neutral. Any non-anomalous linear combination

$$
R_{t}=R_{0}+\sum_{I} s_{I} F_{I}
$$


is a candidate $R$-symmetry in the sense that the SUSY generators have the appropriate charge under $R_{t}$ (' $t$ ' stands for 'trial'). However, only one is the distinguished $R$-symmetry appearing in the superconformal group. In simple cases, we can identify the correct linear combination from symmetry considerations alone. The prototypical example is supersymmetric QCD with $\frac{3}{2} N_{c}<N_{f}<3 N_{c}$ and zero superpotential, which flows to a conformal fixed point in the IR, and has only a single non-anomalous $R=R_{t}$ that commutes with all flavor symmetries. In the UV, there is nothing special about this $R$, but in the deep IR, it becomes part of the emergent $\mathrm{SU}(2,2 \mid 1)$ superconformal symmetry and determines the scaling dimensions of chiral operators.

Theories with less symmetry generally have a non-trivial affine space of possible $R_{t}$ 's. However, a simple condition [15] uniquely determines the correct superconformal $R$-charge: it is a local maximum of

$$
a\left(R_{t}\right)=\frac{3}{32}\left[3 \operatorname{Tr}\left(R_{t}^{3}\right)-\operatorname{Tr}\left(R_{t}\right)\right],
$$

where $\operatorname{Tr}\left(R_{t}^{3}\right)$ and $\operatorname{Tr}\left(R_{t}\right)$ are the coefficients of the gauge anomaly $\left\langle\partial_{\mu} J_{R_{t}}^{\mu} J_{R_{t}}^{\nu} J_{R_{t}}^{\rho}\right\rangle$ and gravitational anomaly $\left\langle\partial_{\mu} J_{R_{t}}^{\mu} T_{\nu \rho} T_{\sigma \gamma}\right\rangle$, respectively. This condition is called ' $a$-maximization.' We review the proof in appendix A.

When our CFT arises as the fixed point of a theory that is weakly coupled in the UV, one can often reliably compute the traces in perturbation theory in the weakly-coupled description using the 't Hooft anomaly-matching conditions. An important exception to this occurs when accidental U(1) symmetries emerge in the IR which aren't manifest in the UV Lagrangian - in this case one must also include these accidental symmetries when maximizing $a\left(R_{t}\right)$.

One possible signal that accidental symmetries are arising is that a gauge-invariant operator $\mathcal{O}$ in the chiral ring appears to violate the bound required by unitarity, given by $R_{\mathcal{O}} \geq 2 / 3$ for scalar operators [39-41]. In this case a reasonable interpretation is that the operator $\mathcal{O}$ is becoming a free field (with $R_{\mathcal{O}}=2 / 3$ ) [38] and that an accidental symmetry associated with rotations of $\mathcal{O}$ is emerging in the IR. A useful description of this phenomenon [42] that makes the emergent symmetry manifest involves introducing an additional vector-like pair of fields $\{L, M\}$ with superpotential

$$
W_{L M}=L(M-\mathcal{O})
$$

When $\mathcal{O}$ is consistent with the unitarity bound, we can think of this as a (somewhat trivial) dual description of the theory we started with. $L$ and $M$ are massive, so we could just integrate them out out. Equivalently, when the operator $L M$ becomes marginal, we have $R(L)=2-R(M)$, which means that the contributions of $L$ and $M$ cancel in the $a$-maximization calculation, so they don't influence the $R$-charges of other fields.

The theory with $L$ and $M$ is presumably identical to the original in the deep IR. But when $\mathcal{O}$ violates the unitarity bound, we can now describe what's going on. The coupling $L M$ flows to zero, leaving us with a free field $M$ with $R$-charge $2 / 3$. Meanwhile, the nonzero coupling $L \mathcal{O}$ sets $\mathcal{O}$ to zero in the chiral ring, resolving any conflict with the unitarity bound. The field $L$ has $R(L)=2-R(\mathcal{O})$, and its contribution to a no longer 
cancels with $M$. Including $L$ and $M$ in the $a$-maximization procedure requires the simple modification $[43,44]$

$$
\begin{aligned}
a\left(R_{t}\right) & \rightarrow a\left(R_{t}\right)+a(M)+a(L) \\
& =a\left(R_{t}\right)+a(2 / 3)-a(\mathcal{O}) \\
& =a\left(R_{t}\right)+\frac{\operatorname{dim}\left(r_{\mathcal{O}}\right)}{96}\left(2-3 R_{\mathcal{O}}\right)^{2}\left(5-3 R_{\mathcal{O}}\right),
\end{aligned}
$$

where $r_{\mathcal{O}}$ denotes the representation of $\mathcal{O}$. It's important to note that $L$ and $M$ can contribute to other anomalies as well. For instance, if $\mathcal{O}$ is in a non-trivial representation of $\mathrm{SU}(5)_{\mathrm{GUT}}$, we must include $L$ and $M$ in calculating $\beta_{g_{5}}$ whenever $\mathcal{O}$ violates the unitarity bound.

Describing this special case of a gauge-singlet operator becoming free is relatively simple. More generally, however, it is not always easy to determine when accidental symmetries arise, and it is important to study carefully any known dual descriptions of the theory under consideration in order to gain evidence for their emergence. See, e.g., [42, 44-53] for additional discussion and many examples.

\subsection{1 $a$-maximization in the toy model}

As a simple example of a-maximization, let us consider the toy model in table 1, with $R_{t}$-charges $R_{X}, R_{\bar{X}}, R_{S}, R_{\bar{S}}, R_{T_{1}}$ for the superfields. $\left(T_{2}, T_{3}\right.$, and the rest of the MSSM do not couple to the strong sector, so they are free fields with $R=2 / 3$.) In the UV, gauge and gravitational anomalies come from the gauginos which have $R_{t}$-charge 1 , and from the matter fermions which have $R_{t}$-charges 1 less than their associated chiral superfields. Thus,

$$
a\left(R_{t}\right)=\frac{3}{32}\left[2\left(N^{2}-1\right)+\sum_{i} \operatorname{dim}\left(r_{i}\right)\left(3\left(R_{i}-1\right)^{3}-\left(R_{i}-1\right)\right)\right],
$$

where the index $i$ runs over all of the chiral superfields, and $r_{i}$ is the representation of each field.

The trial $\mathrm{U}(1)_{\mathrm{R}}$ should be anomaly-free with respect to $G$, so we have the constraint

$$
\begin{aligned}
0 & =T(G)+\sum_{i}\left(R_{i}-1\right) T\left(r_{i}\right) \\
& =N+5\left(R_{X}-1\right)+5\left(R_{\bar{X}}-1\right)+\frac{1}{2}\left(R_{S}-1\right)+\frac{1}{2}\left(R_{\bar{S}}-1\right),
\end{aligned}
$$

where $T(r)$ is the Dynkin index of $r$ as a $G$-representation. Using $\frac{3}{2} R_{i}=1+\frac{\gamma_{i}}{2}$, we see that (2.21) is equivalent to vanishing of the numerator of the exact NSVZ $\beta$-function [54-57]

$$
\beta_{g_{G}}=\frac{-\left(3 T(G)-\sum_{i}\left(1-\gamma_{i}\right) T\left(r_{i}\right)\right)}{16 \pi^{2}\left(1-\frac{T(G) g_{G}^{2}}{8 \pi^{2}}\right)} g_{G}^{3}
$$

That is, $R$ is conserved if and only if the theory is conformal, which reflects the fact that supersymmetry relates the $R$-current anomaly $\partial_{\mu} J_{R}^{\mu}$ to the trace of the stress-energy tensor $T_{\mu}^{\mu}$. 


\begin{tabular}{|c|c|c|c|c|c|}
\hline$N$ & $R_{T_{1}}$ & $R_{X}$ & $R_{\bar{X}}$ & $R_{S}$ & $R_{\bar{S}}$ \\
\hline 4 & .686 & .632 & .637 & .677 & .632 \\
5 & .771 & .683 & .546 & .533 & .533 \\
6 & .920 & .625 & .455 & .439 & .439 \\
7 & 1.191 & .445 & .364 & .356 & .356 \\
\hline
\end{tabular}

Table 2. $R$-charges of our toy model with one light generation (table 1).

A second constraint on $R_{t}$ comes from the fact that the coupling $T_{1} \bar{X} S$ is exactly marginal in our CFT. Thus, we have

$$
2=R_{T_{1}}+R_{\bar{X}}+R_{S}
$$

Finally, numerically minimizing (2.20) subject to our two constraints yields the $R$-charges in table 2. Note that $R_{T_{1}}$ increases with $N$, reflecting the fact that supersymmetric QCD becomes more strongly coupled as $N \rightarrow 2 N_{f} / 3$, pulling the anomalous dimensions of the exotic quarks more negative. We could alternatively analyze our theory in a Seiberg dual description where the coupling $T_{1} \bar{X} S$ becomes a mass coupling to a meson $T_{1} M_{\bar{X} S}$. The magnetic description becomes more weakly-coupled as $N \rightarrow 2 N_{f} / 3$, and $R_{M_{\bar{X} S}}$ approaches its free value $2 / 3$. The $a$-maximization procedure is identical in this dual description, since the two theories have matching anomalies. We present further discussion of the dual description in appendix B.

\section{$3 \quad$ Vector-like models}

In this section we will attempt to find the simplest superconformal models that display a realistic flavor structure. For simplicity we will primarily consider conformal sectors that are vector-like. This can easily ensure that the conformal sector exits at a high scale and that all exotic states decouple, because one can simply write down (large) mass terms for all of the fields. ${ }^{2}$ For example, in the toy model discussed in the previous section we can write down the mass terms $W \supset m_{X} X \bar{X}+m_{S} S \bar{S}$. Assuming that $m_{X}$ becomes marginal first, we expect the CFT regime to exit at a scale $\mu \sim \Lambda_{c}$, where $\Lambda_{c}=m_{X}\left(\Lambda_{c} / \Lambda\right)^{\frac{1}{2}\left(\gamma_{X}+\gamma_{\bar{X}}\right)}$. Meanwhile, below the scale $\mu \sim m_{S}\left(\Lambda_{c} / \Lambda\right)^{\frac{1}{2}\left(\gamma_{S}+\gamma_{\bar{S}}\right)}$ all exotic states will have decoupled and we are left with the MSSM at low energies.

Furthermore, we will mainly consider theories with an $\mathrm{SU}(5)_{\text {GUT }}$ symmetry given their many successful predictions. We will attempt to remain agnostic about the details of GUTbreaking and doublet-triplet splitting, as these issues are (for the most part) decoupled. As discussed in section 2.1, the minimal quasi-realistic structure in the context of SU $(5)_{\text {GUT }}$ is that of a '10-centered' flavor model, or a model that generates different suppression factors for two matter fields in the 10-representation. We will start by finding models in this class.

\footnotetext{
${ }^{2}$ By contrast, [6] focused on chiral strong sectors, with the CFT-breaking scale $\Lambda_{c}$ generated dynamically by some additional gauge group. This is a promising way to avoid a hierarchy problem for $\Lambda / \Lambda_{c}$, but the models are more complicated and there is no general guarantee that exotic states will be massive.
} 
In several cases, the models can be extended easily to generate suppression factors for one or more generations of $\overline{\mathbf{5}}$ 's, or possibly the $\overline{\mathbf{5}}$ Higgs.

As a minimum requirement, the conformal sector should then contain two singlet operators $\mathcal{O}_{1,2}$ in the $\overline{\mathbf{1 0}}$ represenation of $\mathrm{SU}(5)_{\mathrm{GUT}}$, so that one can introduce couplings

$$
W_{\text {int }}=T_{1} \mathcal{O}_{1}+T_{2} \mathcal{O}_{2} .
$$

These interactions should be marginal couplings of the CFT in order for $T_{1,2}$ to acquire large anomalous dimensions, and $\mathcal{O}_{1}$ and $\mathcal{O}_{2}$ should have different dimensions under the CFT in order to explain the hierarchy. In particular, there should be no symmetry relating them. On the other hand, we would also like to have evidence that the CFT exists and that there is a reasonable flow that could lead to the desired fixed point. To this end, we would like these operators to be relevant deformations of the CFT in which they are absent. Typically this requires that the coupling $T_{i} \mathcal{O}_{i}$ involve at most three or four fields. Of course, CFT gauge interactions can lower the dimensions of strong-sector fields, but in many models one will leave the conformal regime or run into violations of the unitarity bound before the 5 -field operators can become relevant.

At what scale should the CFT exit? One strong constraint arises if the interactions in eq. (3.1) violate baryon and lepton number. This will be the case in the simplest models that we will consider. In this situation integrating out the CFT states around the scale $\Lambda_{c}$ will then induce dimension 6 proton-decay operators in the Kähler potential suppressed by $\sim 16 \pi^{2} /\left(N \Lambda_{c}^{2}\right)$. This implies that $\Lambda_{c}$ should be near or above the GUT scale in these models. ${ }^{3}$ There is then a rather small allowed window for conformal running,

$$
M_{\mathrm{GUT}} \lesssim \Lambda_{c}<\Lambda \lesssim M_{\mathrm{pl}}
$$

which in turn means that the theory will need to be fairly strongly-coupled in order to reproduce the observed hierarchies.

Since the CFT sector will introduce many new fields in representations of SU $(5)_{\text {GUT }}$, an additional strong constraint on these models comes from requiring that the GUT gauge coupling remain perturbative throughout the conformal window. Since some of these fields will have large anomalous dimensions, we should integrate the full NSVZ $\beta$-function eq. (2.22), which we will parameterize as

$$
\beta_{g_{5}}=\frac{\mathcal{A}}{16 \pi^{2}\left(1-\frac{5 g_{5}^{2}}{8 \pi^{2}}\right)} g_{5}^{3},
$$

where

$$
\mathcal{A} \equiv-3 \operatorname{Tr}\left[\mathrm{U}(1)_{\mathrm{R}} \mathrm{SU}(5)_{\mathrm{GUT}}^{2}\right]=-15+\sum_{i}\left(1-\gamma_{i}\right) T\left(r_{i}\right)
$$

It is clear that we can only determine the evolution of $g_{5}$ after we know the correct $\mathrm{U}(1)_{\mathrm{R}}$ symmetry, which can in turn only be determined by performing $a$-maximization. However,

\footnotetext{
${ }^{3}$ On the other hand, it is interesting to note that superconformal flavor models can actually improve the situation with dimension 5 proton-decay operators, because they will also receive suppression from the $\epsilon_{i}$ factors. We refer readers to [6] for a more thorough discussion of these operators.
} 


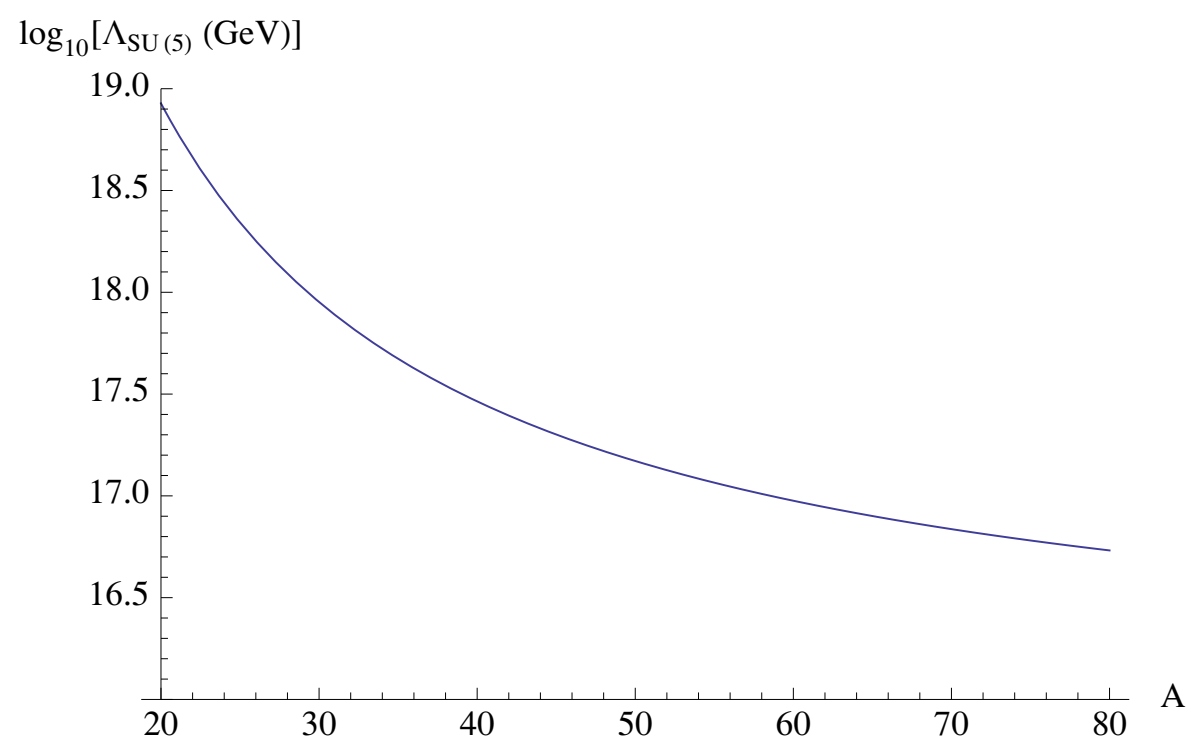

Figure 1. Location of the $\mathrm{SU}(5)_{\text {GUT }}$ Landau pole as a function of the $\beta_{g_{5}}$-numerator $\mathcal{A}$, assuming that all new matter enters at $\Lambda_{c} \sim 10^{16} \mathrm{GeV}$.

if we assume that the CFT exits and all exotic states charged under $\mathrm{SU}(5)_{\mathrm{GUT}}$ decouple near $\Lambda_{c} \sim 10^{16} \mathrm{GeV}$, we can still place a model-independent bound on $\Lambda$ as a function of $\mathcal{A}$.

Integrating eq. (3.3) gives a Landau pole $\Lambda_{\mathrm{SU}(5)}$ at

$$
\log \frac{\Lambda_{\mathrm{SU}(5)}}{\Lambda_{c}}=\frac{5}{\mathcal{A}}\left(\frac{8 \pi^{2}}{5 g_{5}^{2}\left(\Lambda_{c}\right)}+\log \frac{5 g_{5}^{2}\left(\Lambda_{c}\right)}{8 \pi^{2}}-1\right)
$$

The suppression factors $\epsilon_{i}$ should be generated well below $\Lambda_{\mathrm{SU}(5)}$, since otherwise the approximation of our theory as a weakly perturbed SCFT breaks down. If we had an appropriate dual description of the $\mathrm{SU}(5)_{\mathrm{GUT}}$ group, it might be possible to make sense of a scenario where conformal running persists through the Landau pole. But we would likely still lose any semblance of a simple, predictive solution to the flavor problem. Thus, we will demand that the conformal running distance $\log \frac{\Lambda}{\Lambda_{c}}$ not exceed that of eq. (3.5). We plot this bound in figure 1 , where we have taken $\alpha_{5}\left(\Lambda_{c}\right)=g_{5}^{2}\left(\Lambda_{c}\right) / 4 \pi=1 / 25$ as determined from running up the low-energy gauge couplings. The curve indicates the location of $\Lambda_{\mathrm{SU}(5)}$. We will find that this constraint rules out a number of otherwise viable models, because they would require $\Lambda$ to be larger than allowed by this bound in order to reproduce the observed hierarchies.

Below we will attempt to construct the simplest realistic models that avoid the above constraints. We will start by considering the simplest extension of the toy model that can accommodate two light generations, but we will find that the constraint from the SU $(5)_{\text {GUT }}$ Landau pole is too strong. This will motivate looking for models containing only $\mathbf{5}$ and $\overline{\mathbf{5}}$ representations of $\mathrm{SU}(5)_{\mathrm{GUT}}$ in order to minimize the contribution to $\beta_{g_{5}}$ from the $\mathrm{CFT}$ sector. We will then analyze the simplest such models based on $\mathrm{SU}(\mathrm{N}), \mathrm{SO}(\mathrm{N})$, and $\mathrm{Sp}(2 \mathrm{~N})$ gauge groups. 


\begin{tabular}{|c|c|c|}
\hline & $\mathrm{SU}(5)_{\mathrm{GUT}}$ & $\mathrm{SU}(\mathrm{N})$ \\
\hline$X+\bar{Q}+S$ & $\mathbf{1 0}+\overline{\mathbf{5}}+\mathbf{1}$ & $\square$ \\
$\bar{X}+Q+\bar{S}$ & $\overline{\mathbf{1 0}}+\mathbf{5}+\mathbf{1}$ & $\bar{\square}$ \\
\hline
\end{tabular}

Table 3. Matter content of the $\mathbf{1 0}+\overline{\mathbf{5}}+\mathbf{1}$ model.

\section{$3.110+\overline{5}+1$ model}

We will start by extending our toy model from section 2.2 to generate suppression factors for two generations. An obvious way to get two couplings to $T_{i}$ 's is to add a second pair of GUT singlets $S^{\prime}+\bar{S}^{\prime} \in(\mathbf{1}, \square) \oplus(\mathbf{1}, \bar{\square})$, giving us another $\mathbf{1 0} \overline{\mathbf{1 0}} \mathbf{1}$ coupling: $T_{2} \bar{X} S^{\prime}$. However, this will not work because there is a symmetry relating $S$ and $S^{\prime}$ that would ensure that $T_{1}$ and $T_{2}$ have the same anomalous dimension. The only other types of 3 -field GUT couplings to $\mathbf{1 0}$ 's are are 10105 and $\mathbf{1 0} \overline{\mathbf{5}} \overline{\mathbf{5}}$. We can get the first of these by adding a $(\mathbf{5}, \bar{\square}) \oplus(\overline{\mathbf{5}}, \square)$ pair, leaving us with the matter content of table 3 .

Under the $\mathrm{SU}(\mathrm{N})$ gauge theory, there are $N_{f}=16$ flavors of vector-like quarks. Thus, the theory with vanishing superpotential is in an interacting conformal regime for $3 N / 2<$ $16<3 N$, or equivalently $6 \leq N \leq 10$. Meanwhile, from the point of view of $\mathrm{SU}(5)_{\mathrm{GUT}}$ we have added $N$ vector-like pairs of generations, and we should be concerned about the constraint from the SU $(5)_{\text {GUT }}$ Landau pole. Indeed we'll find shortly that this model cannot account for the observed hierarchy if $g_{5}$ remains perturbative in the conformal window.

In addition to the interactions $T_{i} X Q$ and $T_{i} \bar{X} S$, this sector admits other couplings to the Standard Model. The ones that are relevant at weak coupling are the 3-field operators $\bar{F}_{i} Q S, \bar{H} Q S$, and $H \bar{Q} \bar{S}$. In addition, a number of 4-field operators can potentially become relevant at strong coupling. However, in order to forbid dangerous dimension 3 and 4 lepton- and baryon-number violating operators, a realistic theory requires an additional approximate (discrete or continuous) symmetry, which may in turn forbid some subset of the allowed deformations. Here we will simply focus on the interactions that are the most interesting for flavor physics, giving possible symmetries (where appropriate) that would forbid the remaining operators.

The minimal superpotential we need for a $\mathbf{1 0}$-centered model is

$$
W_{\mathrm{int}}=T_{1} X Q+T_{2} \bar{X} S
$$

As before, without loss of generality we can simply define the linear combinations of matter fields that couple to the above CFT operators to be $T_{1}$ and $T_{2}$. If the theory has, e.g., an approximate $\mathrm{U}(1)_{\mathrm{R}}$ symmetry in the UV under which $\{H, \bar{H}, Q, S\}$ have charge 0 , $\left\{T_{i}, \bar{F}_{i}, N_{i}, X, \bar{X}\right\}$ have charge 1 , and $\{\bar{Q}, \bar{S}\}$ have charge 2 , then these are the only allowed interactions. ${ }^{4}$

\footnotetext{
${ }^{4}$ Note that this is similar to the symmetry proposed in [58], which was in part motivated by a possible solution to the doublet-triplet splitting problem. This symmetry is not to be confused with the superconformal $\mathrm{U}(1)_{\mathrm{R}}$ that becomes important during conformal running. Rather, here we are imagining that the theory above the conformal regime has an approximate symmetry that causes some operators to have smaller coefficients than others.
} 


\begin{tabular}{|c|c|c|c|c|c|c|c|c|c|c|c|}
\hline$N$ & $R_{T_{1}}$ & $R_{T_{2}}$ & $R_{X}$ & $R_{\bar{Q}}$ & $R_{S}$ & $R_{\bar{X}}$ & $R_{Q}$ & $R_{\bar{S}}$ & $\mathcal{A}$ & $\Lambda_{\mathrm{SU}(5)} / \Lambda_{c}$ & $\Lambda / \Lambda_{c}$ \\
\hline 6 & .740 & .706 & .625 & .616 & .673 & .621 & .635 & .616 & 23.6 & $10^{2.48}$ & $10^{22.91 \pm 4.33}$ \\
7 & .862 & .782 & .561 & .546 & .661 & .557 & .576 & .546 & 32.6 & $10^{1.80}$ & $10^{8.60 \pm 1.63}$ \\
8 & .992 & .885 & .497 & .483 & .620 & .495 & .511 & .483 & 42.9 & $10^{1.37}$ & $10^{4.96 \pm 0.77}$ \\
9 & 1.123 & 1.021 & .434 & .425 & .544 & .435 & .443 & .425 & 54.4 & $10^{1.08}$ & $10^{3.26 \pm 0.27}$ \\
10 & 1.251 & 1.196 & .373 & .369 & .429 & .375 & .377 & .369 & 67.2 & $10^{0.87}$ & $10^{2.35 \pm 0.01}$ \\
\hline
\end{tabular}

Table 4. $R$-charges in the $\mathbf{1 0}+\overline{\mathbf{5}}+\mathbf{1}$ model with the superpotential $W=T_{1} X Q+T_{2} \bar{X} S$ assumed to be marginal. The last three columns give: the $\mathrm{SU}(5)_{\mathrm{GUT}}$ anomaly $\mathcal{A}$, the position of the $\mathrm{SU}(5)_{\mathrm{GUT}}$ Landau pole assuming $\alpha_{5}\left(\Lambda_{c}\right)=1 / 25$, and the phenomenologically required size for the conformal window. We have assumed the presence of an additional GUT-breaking adjoint above $\Lambda_{c}$.

Now we will determine the $\mathrm{U}(1)_{\mathrm{R}}$ symmetry using $a$-maximization. The superpotential eq. (3.6) and anomaly cancelation impose the constraints

$$
\begin{aligned}
& 2=R_{T_{1}}+R_{X}+R_{Q} \\
& 2=R_{T_{2}}+R_{\bar{X}}+R_{S} \\
& 0=N+5\left(R_{X}-1\right)+5\left(R_{\bar{X}}-1\right)+\frac{5}{2}\left(R_{Q}-1\right)+\frac{5}{2}\left(R_{\bar{Q}}-1\right)+\frac{1}{2}\left(R_{S}-1\right)+\frac{1}{2}\left(R_{\bar{S}}-1\right) .
\end{aligned}
$$

Maximizing $a\left(R_{t}\right)$ subject to these constraints then yields the $R$-charges given in table 4 .

In the table (and throughout the paper), we have defined the phenomenologically required conformal window $\Lambda / \Lambda_{c}$ to be the running distance required to generate suppression factors that are simultaneously within a factor of 3 of the values $\epsilon_{T_{1}}=.003$ and $\epsilon_{T_{2}}=.04$. The window on $\epsilon_{T_{1}}$ covers both the somewhat smaller suppression factor preferred by the up quark mass, as well as the somewhat larger suppression factor preferred by the Cabibbo angle. In addition, we've indicated the numerator of $\beta_{g_{5}}$ and the position of the $\mathrm{SU}(5)_{\mathrm{GUT}}$ Landau pole assuming $\alpha_{5}\left(\Lambda_{c}\right)=1 / 25$, as would occur for $\Lambda_{c} \sim M_{\mathrm{GUT}}$. Also throughout this paper we have assumed that there is an extra GUT-breaking adjoint in the spectrum above $M_{\mathrm{GUT}}$, which gives a contribution to $\beta_{g_{5}}$ in addition to that from the exotic sector and the usual matter fields. This is a conservative assumption in that most realistic GUTbreaking sectors will require at least this much additional matter. However, if a clever way could be found to combine the physics of GUT breaking and conformal symmetry breaking, it is possible that the bounds could be somewhat relaxed.

In all cases, the required $\Lambda$ is larger than the Landau pole $\Lambda_{\mathrm{SU}(5)}$. One might try to avoid this fate by adding additional relevant deformations to the superpotential. For instance, when $N \geq 8$, the operator $(\bar{Q} Q)^{2}$ is gauge invariant and relevant, so one could impose the constraint that it too becomes marginal. However, we have not found a set of relevant deformations that can save this model.

It's striking that such a simple model so badly violates $\Lambda<\Lambda_{\mathrm{SU}(5)}$. A natural question to ask is whether there exist any simple vector-like models that can avoid this bound. In appendix $\mathrm{C}$ we have listed all qualitatively different simple group models containing two distinct couplings to the $T_{i}$ 's involving three or four fields. Looking through this list, we find only two other models that possess two three-field couplings. The first is similar to 


\begin{tabular}{|l|c|c|}
\hline & $\mathrm{SU}(5)_{\mathrm{GUT}}$ & $\mathrm{SU}(\mathrm{N})$ \\
\hline$Q_{1}+\bar{Q}_{2}$ & $\mathbf{5}+\overline{\mathbf{5}}$ & $\square$ \\
$\bar{Q}_{1}+Q_{2}$ & $\overline{\mathbf{5}}+\mathbf{5}$ & $\square$ \\
$A$ & $\mathbf{1}$ & Ad. \\
\hline
\end{tabular}

Table 5. CFT sector based on $\mathrm{SU}(\mathrm{N})$ with an adjoint.

the present model and based on $\mathrm{SU}(\mathrm{N})$ with fundamentals in the $\mathbf{1 0}+\mathbf{5}+\overline{\mathbf{5}}$ representation and anti-fundamentals in the $\overline{\mathbf{1 0}}+\overline{\mathbf{5}}+\mathbf{5}$ representation. The second is based on $\operatorname{Sp}(2 \mathrm{~N})$ with fundamentals in the $\mathbf{1 0}+\overline{\mathbf{1 0}}+\mathbf{5}+\overline{\mathbf{5}}$. However, we find that both of these models have similar problems with the $\mathrm{SU}(5)_{\text {GUT }}$ Landau pole. To go forward, it is clear that we need to additionally consider models with four-field couplings.

In fact, it appears likely that almost any vector-like CFT sector containing chiral superfields $X$ in 10's and $\overline{\mathbf{1 0}}$ 's of SU(5) GUT will be problematic when one considers $\beta_{g_{5}}$. The reason is that we typically need the CFT to have a relatively large gauge group $G$ in order to have a sufficiently strongly-coupled fixed point. However, since the fields $X$ transform in representations of $G$, they look like a large number of SM $\mathbf{1 0}+\overline{\mathbf{1 0}}$ pairs, each of which contributes roughly a factor of 3 (as opposed to 1 for $\mathbf{5}+\overline{\mathbf{5}}$ pairs) to the anomaly coefficient $\mathcal{A}$. Further, strong gauge interactions tend to drive $\gamma_{X}$ negative, which only increases the contribution to $\beta_{g_{5}}$. These considerations motivate us to focus on CFT sectors that contain only GUT $\mathbf{5}$ 's, $\overline{\mathbf{5}}$ 's, and singlets. This cuts down the space of models considerably, and there are only a few that we need to consider.

As summarized in appendix C, we find exactly six possible model structures. Two of the models require baryonic operators that are specific to $\mathrm{SU}(3)$ and $G_{2}$, respectively. However, we find that these models are too weakly coupled to be phenomenologically successful and will not discuss them further. The remaining models are based on $\mathrm{SU}(\mathrm{N})$ with an adjoint, $\mathrm{SO}(\mathrm{N})$ with an adjoint or symmetric tensor, and $\mathrm{Sp}(2 \mathrm{~N})$ with an anti-symmetric tensor. We will consider these models and their deformations below, and ultimately find that both the $\mathrm{SU}(\mathrm{N})$ models and the $\mathrm{Sp}(2 \mathrm{~N})$ models can potentially be phenomenologically successful.

\section{$3.2 \mathrm{SU}(\mathrm{N})$ with an adjoint}

Next we will consider perhaps the simplest candidate CFT based on a strong SU(N) gauge group that does not contain any $\overline{\mathbf{1 0}}$ representations of $\mathrm{SU}(5)_{\mathrm{GUT}}$. In order to allow for two distinct couplings to the Standard Model 10's, the sector contains an SU(N) adjoint $A$ in addition to vector-like pairs of fundamentals in the $\mathbf{5}+\overline{\mathbf{5}}$ representation of $\mathrm{SU}(5)_{\text {GUT }}$. The matter content is given in table 5 . Without a superpotential, the $\mathrm{SU}(\mathrm{N})$ theory is simply adjoint SQCD with $N_{f}=10$, which is believed to flow to an interacting conformal fixed point for all $0<N_{f}<2 N$ [59], or equivalently $N \geq 6$.

The two lowest dimension couplings to 10's are

$$
W_{\text {int }}=T_{1} \bar{Q}_{1} \bar{Q}_{2}+T_{2} \bar{Q}_{1} A \bar{Q}_{2} .
$$

As it stands, this theory doesn't admit any other three- or four-field couplings to the Standard Model. However, if desired it would be straightforward to introduce additional 


\begin{tabular}{|c|c|c|c|c|c|c|c|c|}
\hline$N$ & $R_{T_{1}}$ & $R_{T_{2}}$ & $R_{Q_{1,2}}$ & $R_{\bar{Q}_{1,2}}$ & $R_{A}$ & $\mathcal{A}$ & $\Lambda_{\mathrm{SU}(5)} / \Lambda_{c}$ & $\Lambda / \Lambda_{c}$ \\
\hline 15 & 1.012 & .670 & .479 & .494 & .342 & 41.6 & $10^{1.41}$ & - \\
16 & 1.030 & .703 & .469 & .485 & .327 & 45.4 & $10^{1.29}$ & - \\
17 & 1.047 & .734 & .460 & .476 & .313 & 49.2 & $10^{1.19}$ & - \\
18 & 1.063 & .763 & .452 & .468 & .300 & 53.1 & $10^{1.10}$ & - \\
19 & 1.079 & .791 & .444 & .461 & .288 & 57.0 & $10^{1.03}$ & - \\
20 & 1.093 & .816 & .437 & .453 & .277 & 61.0 & $10^{0.96}$ & $10^{4.40 \pm 0.29}$ \\
21 & 1.107 & .840 & .431 & .446 & .267 & 65.0 & $10^{0.90}$ & $10^{4.04 \pm 0.50}$ \\
22 & 1.120 & .862 & .425 & .440 & .258 & 69.0 & $10^{0.85}$ & $10^{3.77 \pm 0.64}$ \\
23 & 1.133 & .883 & .419 & .434 & .249 & 73.1 & $10^{0.80}$ & $10^{3.61 \pm 0.68}$ \\
24 & 1.145 & .903 & .414 & .428 & .241 & 77.2 & $10^{0.76}$ & $10^{3.52 \pm 0.67}$ \\
25 & 1.156 & .922 & .409 & .422 & .234 & 81.3 & $10^{0.72}$ & $10^{3.44 \pm 0.65}$ \\
26 & 1.166 & .940 & .404 & .417 & .227 & 85.5 & $10^{0.69}$ & $10^{3.37 \pm 0.64}$ \\
\hline
\end{tabular}

Table 6. $R$-charges in the $\mathrm{SU}(\mathrm{N})$ adjoint model with superpotential $W=T_{1} \bar{Q}_{1} \bar{Q}_{2}+T_{2} \bar{Q}_{1} A \bar{Q}_{2}$ assumed to be marginal. For $N>15$ the operator $\operatorname{Tr}\left[A^{2}\right]$ violates the unitarity bound and the effect of this operator becoming a free field is included in the $a$-maximization procedure. The last three columns give: the $\mathrm{SU}(5)_{\mathrm{GUT}}$ anomaly $\mathcal{A}$, the position of the $\mathrm{SU}(5)_{\mathrm{GUT}}$ Landau pole assuming $g_{5}\left(\Lambda_{c}\right)=0.7$, and the phenomenologically required size for the conformal window.

couplings to $\overline{5}$ 's by adding extra SM singlet flavors to the theory.

Assuming that the terms in eq. (3.8) are the only marginal interactions, it is then straightforward to determine the $\mathrm{U}(1)_{\mathrm{R}}$ symmetry of this theory using $a$-maximization. The $R$-charges are constrained by the superpotential and anomaly cancelation as

$$
\begin{aligned}
& 2=R_{T_{1}}+R_{\bar{Q}_{1}}+R_{\bar{Q}_{2}} \\
& 2=R_{T_{2}}+R_{\bar{Q}_{1}}+R_{A}+R_{\bar{Q}_{2}} \\
& 0=N+\frac{5}{2}\left(R_{Q_{1}}-1\right)+\frac{5}{2}\left(R_{Q_{2}}-1\right)+\frac{5}{2}\left(R_{\bar{Q}_{1}}-1\right)+\frac{5}{2}\left(R_{\bar{Q}_{2}}-1\right)+N\left(R_{A}-1\right) .
\end{aligned}
$$

Maximizing $a\left(R_{t}\right)$ subject to these constraints then gives the $R$-charges in table 6 . It is important to note that $N \geq 15$ is required in order for the second superpotential coupling to be marginal in the CFT. This is because for smaller values of $N$ we would find a violation of the unitarity bound $R_{T_{2}}<2 / 3$, indicating that the coupling must flow to zero. On the other hand, for all $N>15$ the gauge-invariant operator $\operatorname{Tr}\left[A^{2}\right]$ has $R<2 / 3$, and we have modified the $a$-maximization procedure as in eq. (2.19) in order to account for this operator becoming a free field.

While we have arbitrarily stopped at $N=26$, it is clear that in all cases the phenomenologically required running distance can not be achieved without hitting an SU(5) GUT Landau pole. Thus, we next consider whether deforming the theory by an additional superpotential term can improve the situation. 


\begin{tabular}{|c|c|c|c|c|c|c|c|c|}
\hline$N$ & $R_{T_{1}}$ & $R_{T_{2}}$ & $R_{Q_{1,2}}$ & $R_{\bar{Q}_{1,2}}$ & $R_{A}$ & $\mathcal{A}$ & $\Lambda_{\mathrm{SU}(5)} / \Lambda_{c}$ & $\Lambda / \Lambda_{c}$ \\
\hline 11 & 1.448 & .781 & .257 & .276 & .667 & 33.884 & $10^{1.73}$ & - \\
12 & 1.572 & .905 & .186 & .214 & .667 & 34.528 & $10^{1.70}$ & - \\
13 & 1.705 & 1.039 & .119 & .147 & .667 & 35.925 & $10^{1.63}$ & $10^{1.79 \pm 0.14}$ \\
14 & 1.849 & 1.182 & .058 & .076 & .667 & 38.080 & $10^{1.54}$ & $10^{1.44 \pm 0.25}$ \\
\hline
\end{tabular}

Table 7. $R$-charges in the deformed $\mathrm{SU}(\mathrm{N})$ adjoint model with superpotential $W=T_{1} \bar{Q}_{1} \bar{Q}_{2}+$ $T_{2} \bar{Q}_{1} A \bar{Q}_{2}+\operatorname{Tr}\left[A^{3}\right]$ assumed to be marginal. For these values of $N$ the operators $Q_{1} Q_{2}, Q_{1} \bar{Q}_{1}, \bar{Q}_{2} Q_{2}$, and the 15 component of $\bar{Q}_{1} \bar{Q}_{2}$ are assumed to be free fields in the $a$-maximization procedure. The last three columns give: the $\mathrm{SU}(5)_{\mathrm{GUT}}$ anomaly $\mathcal{A}$, the position of the $\mathrm{SU}(5)_{\mathrm{GUT}}$ Landau pole assuming $\alpha_{5}\left(\Lambda_{c}\right)=1 / 25$, and the phenomenologically required size for the conformal window.

\subsubsection{Deformation by $\operatorname{Tr}\left[A^{3}\right]$}

The simplest such deformation consists of the superpotential

$$
W_{\mathrm{int}}=T_{1} \bar{Q}_{1} \bar{Q}_{2}+T_{2} \bar{Q}_{1} A \bar{Q}_{2}+\operatorname{Tr}\left[A^{3}\right] .
$$

This imposes the additional constraint that $R_{A}=2 / 3$. In addition, it is well known [60] that without the couplings to $T_{1,2}$ this theory only flows to an interacting fixed point for $N_{f}>2 N / 3$, or equivalently $N<15$. On the other hand, we find that $R_{T_{2}}>2 / 3$ requires $N>10$, and thus we only need to consider a small range of $N$.

In table 7 we give the result of $a$-maximization for the theory with this deformation. ${ }^{5}$. For each $10<N<15$ we find that the operators $Q_{1} Q_{2}, Q_{1} \bar{Q}_{1}, \bar{Q}_{2} Q_{2}$, and $\bar{Q}_{1} \bar{Q}_{2}$ have $R<2 / 3$, and hence most of these operators must become free fields. An important subtlety is that the $\overline{\mathbf{1 0}}$ component of $\bar{Q}_{1} \bar{Q}_{2}$ is set to zero by the $T_{1}$ equation of motion, and is not part of the chiral ring of the theory. Because of this, the unitarity bound does not apply to this operator, and we should not include it when modifying $a$ to account for the accidental symmetries.

Here we see that the situation is improved for $N=13,14$, since the $\mathrm{SU}(5)_{\text {GUT }}$ Landau pole can potentially occur above the top of the conformal window, given our assumptions. However, since $g_{5}$ is becoming fairly strongly coupled at the top of the conformal window and the running distance is so short, one might worry that our approximation of treating the $\mathrm{SU}(5)_{\mathrm{GUT}}$ as a flavor group is not very good. The tension could be eased somewhat if $\alpha_{5}\left(\Lambda_{c}\right)$ were smaller than the unified value of $1 / 25$, or if we could find a way to use composites of the CFT sector in order to break the GUT group rather than introducing an additional SU(5) GUT adjoint as we have assumed. Nevertheless, we are motivated to see if we can find any models where these tensions can be avoided.

We could now proceed by considering alternative deformations such as $\operatorname{Tr}\left[A^{4}\right]$ or $Q_{i} \bar{Q}_{i} Q_{j} \bar{Q}_{j}$. However, we have not found any simple deformations that can significantly ease these tensions. The root of the problem is that there is simply still too much matter in the CFT sector charged under $\mathrm{SU}(5)_{\text {GUT }}$ relative to how strongly coupled the theory is.

\footnotetext{
${ }^{5}$ We are indebted to Nathaniel Craig for noticing that we mistakenly included incorrect values of $\mathcal{A}$ and $\Lambda_{\mathrm{SU}(5)} / \Lambda_{c}$ in a previous version of this table. This led us to prematurely conclude that the model was not viable. For further discussion of this model and its variations, see [74].
} 


\begin{tabular}{|l|c|c|}
\hline & $\mathrm{SU}(5)_{\mathrm{GUT}}$ & $\mathrm{Sp}(2 \mathrm{~N})$ \\
\hline$Q+\bar{Q}$ & $\mathbf{5}+\overline{\mathbf{5}}$ & $\square$ \\
$A$ & $\mathbf{1}$ & $\boxminus$ \\
\hline
\end{tabular}

Table 8. Matter content of the $\operatorname{Sp}(2 \mathrm{~N})$ model.

Thus, we are motivated to try to find CFT sectors that are even more efficient - we wish to minimize the matter content of the CFT in order to stay deep within the conformal window while still allowing two couplings to Standard Model 10's.

There are two remaining classes of vector-like models that do not contain GUT 10's that we need to consider. The first is based on $\mathrm{SO}(\mathrm{N})$ with an adjoint (or a symmetric tensor) and two fundamental $\mathbf{5}+\overline{\mathbf{5}}$ pairs, which can be thought of as simply taking a subgroup of the present model. However, it is easy to see that this model will be significantly worse than the $\mathrm{SU}(\mathrm{N})$ version. The reason is that an adjoint of $\mathrm{SO}(\mathrm{N})$ has Dynkin index $N-2$, which scales like $N$ just as in $\mathrm{SU}(\mathrm{N})$. However, fundamentals of $\mathrm{SO}(\mathrm{N})$ have index 1 rather than $1 / 2$ for $\mathrm{SU}(\mathrm{N})$, and since there are the same number of fundamentals as in the $\mathrm{SU}(\mathrm{N})$ model $N$ will need to be roughly twice as large to get to the same part of the conformal window. The contribution to the $\mathrm{SU}(5)_{\mathrm{GUT}} \beta$-function is then roughly twice as large, and the model is quickly ruled out.

The final model is based on $\mathrm{Sp}(2 \mathrm{~N})$ with an anti-symmetric tensor and fundamentals in the $\mathbf{5}+\overline{\mathbf{5}}$ representation. The biggest advantage of this model is that it only needs half as many fundamentals in order to introduce couplings to the $T_{i}$ 's, and is hence a good candidate in our search for a more efficient model. In the following section we will proceed to study this model and its possible deformations in more detail.

\section{3 $\mathrm{Sp}(2 \mathrm{~N})$ with an Anti-symmetric Tensor}

The matter content of the $\mathrm{Sp}(2 \mathrm{~N})$ model is summarized in table 8. In order for the theory to be IR-interacting, we must have $N \geq 4$ [61]. The two lowest dimension couplings to the Standard Model are

$$
W_{\mathbf{1 0}}=T_{1} \bar{Q} \bar{Q}+T_{2} \bar{Q} A \bar{Q} .
$$

There are no three- or four-field couplings to $\overline{\mathbf{5}}$ 's, though we will later consider an extension of this model which can couple to $\bar{H}$ and $\bar{F}_{i}$.

Now we will determine the superconformal $U(1)_{R}$ symmetry, assuming $W_{\mathbf{1 0}}$ contains the only marginal interactions. The superpotential and anomaly cancelation impose the constraints

$$
\begin{aligned}
& 2=R_{T_{1}}+2 R_{\bar{Q}} \\
& 2=R_{T_{2}}+2 R_{\bar{Q}}+R_{A} \\
& 0=2(N+1)+5\left(R_{Q}-1\right)+5\left(R_{\bar{Q}}-1\right)+2(N-1)\left(R_{A}-1\right) .
\end{aligned}
$$

Performing $a$-maximization gives the $R$-charges listed in table 9 , where we have arbitrarily stopped at $N=10$. This model can evade the bound $\Lambda<\Lambda_{\mathrm{SU}(5)}$ when $N=5,6,7$ and 8 


\begin{tabular}{|c|c|c|c|c|c|c|c|c|}
\hline$N$ & $R_{T_{1}}$ & $R_{T_{2}}$ & $R_{Q}$ & $R_{\bar{Q}}$ & $R_{A}$ & $\mathcal{A}$ & $\Lambda_{\mathrm{SU}(5)} / \Lambda_{c}$ & $\Lambda / \Lambda_{c}$ \\
\hline 4 & 1.045 & .778 & .401 & .477 & .268 & 8.255 & $10^{7.09}$ & - \\
5 & 1.103 & .872 & .382 & .448 & .231 & 11.662 & $10^{5.02}$ & $10^{3.85 \pm 0.73}$ \\
6 & 1.154 & .950 & .369 & .423 & .204 & 15.277 & $10^{3.83}$ & $10^{3.45 \pm 0.65}$ \\
7 & 1.197 & 1.014 & .359 & .401 & .183 & 19.076 & $10^{3.07}$ & $10^{3.09 \pm 0.51}$ \\
8 & 1.234 & 1.067 & .351 & .383 & .166 & 23.025 & $10^{2.54}$ & $10^{2.76 \pm 0.34}$ \\
9 & 1.263 & 1.111 & .344 & .368 & .152 & 27.076 & $10^{2.16}$ & $10^{2.55 \pm 0.26}$ \\
10 & 1.288 & 1.147 & .338 & .356 & .140 & 31.215 & $10^{1.88}$ & $10^{2.40 \pm 0.20}$ \\
\hline
\end{tabular}

Table 9. $R$-charges in the $\operatorname{Sp}(2 \mathrm{~N})$ model with the superpotential $W_{\mathbf{1 0}}=T_{1} \bar{Q} \bar{Q}+T_{2} \bar{Q} A \bar{Q}$ assumed to be marginal. When the operators $\operatorname{Tr}\left[A^{k}\right]$ violate the unitarity bound they are assumed to become free fields and the resulting accidental symmetry is included in the $a$-maximization procedure.

(but the constraint is too strong at larger values). However, the required running distance in all of these cases is $\Lambda / \Lambda_{c} \gtrsim M_{\mathrm{pl}} / M_{\mathrm{GUT}} \sim 10^{2}$ or 3 . It is not necessarily fatal to have the upper end of the conformal window near $M_{\mathrm{pl}}$. However if $\Lambda \gtrsim M_{\mathrm{pl}}$, we lose confidence in our naïve calculation of Standard Model wave-function factors, since Planck-scale matter will likely influence the anomalous dimensions. The case of $N=8$ is perhaps the best behaved in this light.

\subsubsection{Deformation by $\operatorname{Tr}\left[A^{k+1}\right]$}

Next, we will analyze possible deformations of our CFT, some of which improve consistency with the bounds $\Lambda<\Lambda_{\mathrm{SU}(5)}$ and $\Lambda<M_{\mathrm{pl}}$. First, we can consider adding $\operatorname{Tr}\left[A^{k+1}\right]$ to the superpotential as a marginal interaction. Note that the theory without $W_{\mathbf{1 0}}$ has a known dual description with gauge group $\operatorname{Sp}\left(2 \mathrm{k}\left(\mathrm{N}_{\mathrm{f}}-2\right)-2 \mathrm{~N}\right)$ [61], where $N_{f}=5$ in our case. This dual magnetic theory is IR free when

$$
N>\left(k-\frac{1}{2}\right) N_{f}-2(k-1)=3 k-\frac{1}{2} .
$$

We expect the same to be true in the theory with $W_{\mathbf{1 0}}$ turned on, since in the dual description (which we will discuss further below) it simply corresponds to deforming the theory by mesonic mass operators. Consequently, our $\operatorname{Tr}\left[A^{k+1}\right]$ deformation engenders an upper limit on values of $N$ for which the theory can have a non-trivial conformal fixed-point.

Let us start by considering the theory deformed by $\operatorname{Tr}\left[A^{3}\right]$, which can have a non-trivial conformal fixed point when $N=4$ or 5 . Performing $a$-maximization gives the $R$-charges in table 10. In the $a$-maximization procedure, we have been careful to take into account the accidental symmetries associated with the operators $Q Q$ and $\bar{Q} Q$ becoming free fields. (Note that we need not include $\bar{Q} \bar{Q}$, as it is zero in the chiral ring and the unitarity bound does not apply.) As discussed in section 2.3, this modifies not only the $a$-maximization procedure, but also the calculation of other anomalies, in particular the numerator of $\beta_{g_{5}}$. As we can see from table 10, this is a dramatic effect. The bounds from the Landau pole $\Lambda_{\mathrm{SU}(5)}$ become much weaker, because the $\{L, M\}$ pairs associated to $Q Q$ and $\bar{Q} Q$ give large negative contributions to $\mathcal{A}$. The theory with $N=5$ is seen to easily evade the Landau pole constraint and fits beautifully between $M_{\mathrm{GUT}}$ and $M_{\mathrm{pl}}$. 


\begin{tabular}{|c|c|c|c|c|c|c|c|c|}
\hline$N$ & $R_{T_{1}}$ & $R_{T_{2}}$ & $R_{Q}$ & $R_{\bar{Q}}$ & $R_{A}$ & $\mathcal{A}$ & $\Lambda_{\mathrm{SU}(5)} / \Lambda_{c}$ & $\Lambda / \Lambda_{c}$ \\
\hline 4 & 1.497 & .830 & .149 & .251 & .667 & 6.063 & $10^{9.66}$ & - \\
5 & 1.786 & 1.119 & .026 & .107 & .667 & 7.163 & $10^{8.18}$ & $10^{1.57 \pm 0.22}$ \\
\hline
\end{tabular}

Table 10. $R$-charges in the $\operatorname{Sp}(2 \mathrm{~N})$ model with the superpotential $W=T_{1} \bar{Q} \bar{Q}+T_{2} \bar{Q} A \bar{Q}+\operatorname{Tr}\left[A^{3}\right]$. assumed to be marginal. Note that the $Q Q$ and $\bar{Q} Q$ operators becoming free results in a significant negative contribution to $\mathcal{A}$.

\begin{tabular}{|c|c|c|c|c|c|c|c|c|}
\hline$N$ & $R_{T_{1}}$ & $R_{T_{2}}$ & $R_{Q}$ & $R_{\bar{Q}}$ & $R_{A}$ & $\mathcal{A}$ & $\Lambda_{\mathrm{SU}(5)} / \Lambda_{c}$ & $\Lambda / \Lambda_{c}$ \\
\hline 4 & 1.331 & .831 & .266 & .334 & .500 & 8.460 & $10^{6.92}$ & - \\
5 & 1.531 & 1.031 & .166 & .234 & .500 & 9.960 & $10^{5.88}$ & $10^{2.00 \pm 0.32}$ \\
6 & 1.787 & 1.287 & .093 & .107 & .500 & 12.409 & $10^{4.72}$ & $10^{1.50 \pm 0.28}$ \\
7 & 2.000 & 1.500 & .000 & .000 & .500 & 13.000 & $10^{4.64}$ & $10^{1.26 \pm 0.23}$ \\
8 & 2.200 & 1.700 & -.100 & -.100 & .500 & 14.200 & $10^{4.24}$ & $10^{1.05 \pm 0.16}$ \\
\hline
\end{tabular}

Table 11. $R$-charges in the $\operatorname{Sp}(2 \mathrm{~N})$ model with the superpotential $W=T_{1} \bar{Q} \bar{Q}+T_{2} \bar{Q} A \bar{Q}+\operatorname{Tr}\left[A^{4}\right]$ assumed to be marginal. Note that when the $Q Q, \bar{Q} Q, Q A Q$, and $\bar{Q} A Q$ operators become free they result in a significant negative contribution to $\mathcal{A}$.

Next let us consider the electric theory with $\operatorname{Tr}\left[A^{4}\right]$ as a marginal interaction, which allows a non-trivial fixed point for $4 \leq N \leq 8$. The results of $a$-maximization are summarized in table 11. We find that the models with $N=5,6,7$, and 8 can also be phenomenologically successful. When the operators $Q Q, \bar{Q} Q, Q A Q$, and $\bar{Q} A Q$ violate the unitarity bound they are assumed to become free fields. Note that we need not do the same for $\bar{Q} \bar{Q}$ and $\bar{Q} A \bar{Q}$ because they are set to zero in the chiral ring and the unitarity bound does not apply. For $N=8$ we see the appearance of negative $R$-charges, which are somewhat unusual. However, we do not see any obvious reason why this theory should be excluded.

We could continue and classify viable models with $k \geq 5$. However, an important point is that the existence of both couplings $T_{1} \bar{Q} \bar{Q}$ and $T_{2} \bar{Q} A \bar{Q}$ in tandem disallows any non-trivial flavor symmetry for $A$. Thus, all couplings $\operatorname{Tr}\left[A^{k}\right]$ are necessarily allowed, and it's perhaps unnatural to expect that our theory should flow to a fixed point with, for instance, $\operatorname{Tr}\left[A^{5}\right]$ as a marginal operator instead of $\operatorname{Tr}\left[A^{3}\right]$. At this point we could also consider deformations by operators like $\bar{Q} Q \bar{Q} Q$, or $\bar{Q} A^{2} Q$. However, we do not find that these lead to a successful phenomenology, and so will not discuss these deformations in detail.

\subsubsection{Dual description}

Here we will briefly discuss the dual description [61] of the theories with a $\operatorname{Tr}\left[A^{k+1}\right]$ superpotential. The matter content of the dual is summarized in table 12, and the interacting superpotential is given by

$$
W_{\text {dual }}=\operatorname{Tr} Y^{k+1}+\sum_{j=1}^{k}\left(M_{Q Q}^{j} \bar{q} Y^{k-j} \bar{q}+M_{\bar{Q} Q}^{j} q Y^{k-j} \bar{q}+M_{\bar{Q} \bar{Q}}^{j} q Y^{k-j} q\right) .
$$




\begin{tabular}{|l|c|c|}
\hline & $\mathrm{SU}(5)_{\mathrm{GUT}}$ & $\mathrm{Sp}(6 \mathrm{k}-2 \mathrm{~N})$ \\
\hline$q+\bar{q}$ & $\mathbf{5}+\overline{\mathbf{5}}$ & $\square$ \\
$Y$ & $\mathbf{1}$ & $\boxminus$ \\
$M_{Q Q}^{j}+M_{\bar{Q} Q}^{j}+M_{\bar{Q} \bar{Q}}^{j}$ & $\mathbf{1 0}+(\mathbf{2 4}+\mathbf{1})+\overline{\mathbf{1 0}}$ & $\mathbf{1}$ \\
\hline
\end{tabular}

Table 12. Matter content of the dual description of the $\operatorname{Sp}(2 \mathrm{~N})$ model deformed by $\operatorname{Tr}\left[A^{k+1}\right]$. The mesons $M_{Q Q}^{j}(j=1, \ldots, k)$ correspond to the operators $Q A^{j-1} Q$ in the electric theory. Similarly, $M_{\bar{Q} Q}^{j} \sim \bar{Q} A^{j-1} Q$ and $M_{\bar{Q} \bar{Q}}^{j} \sim \bar{Q} A^{j-1} \bar{Q}$.

After adding $W_{\mathbf{1 0}}=T_{1} M_{\bar{Q} \bar{Q}}^{1}+T_{2} M_{\bar{Q} \bar{Q}}^{2}$ to the magnetic theory, we could consider integrating out the massive pairs $\left\{T_{1}, M_{\bar{Q} \bar{Q}}^{1}\right\}$ and $\left\{T_{2}, M_{\bar{Q} \bar{Q}}^{2}\right\}$ to get a description with fewer degrees of freedom. However, including the relevant deformations $W_{\text {exit }}=\operatorname{Tr} M_{\bar{Q} Q}^{1}+\operatorname{Tr} Y^{2}$ and expanding the theory around its supersymmetric vacua will induce additional mass operators for the mesons, and in general there will be linear combinations of $T_{1,2}$ with $M_{Q Q}^{j}$ that get lifted. This can introduce additional (incalculable) mixing angles into the Yukawa couplings, which we are here assuming are $O(1)$. We present further discussion of these issues in appendix B.

It is also simple to describe operators becoming free in the dual description. If the full superpotential eq. (3.14) were marginal, we would find that the gauge singlet operators $M_{Q Q}^{1}$ and $M_{\bar{Q} Q}^{1}$ violate the unitarity bound. Since their $R$-charges cannot drop below 2/3, the couplings $M_{Q Q}^{1} \bar{q} Y^{k-1} \bar{q}$ and $M_{\bar{Q} Q}^{1} q Y^{k-1} \bar{q}$ must become irrelevant and flow to zero. Similar considerations apply to the couplings of $M_{Q Q}^{2}$ and $M_{\bar{Q} Q}^{2}$ for $N=7,8$ in the case of $k=3$. Leaving out these couplings and performing $a$-maximization in the dual theory gives $R$-charges and $\mathcal{A}$ in agreement with tables 10 and 11. From this point of view the small values of $\mathcal{A}$ are not surprising, since the theory has only a few GUT multiplets, and many of their $R$-charges are at or near their free values.

\subsubsection{Coupling to $\overline{5}$ 's}

As reviewed in section 2.1, a 10-centered model (with suppression factors only for $T_{1}$ and $T_{2}$ ) is possible at large $\tan \beta$, perhaps given some lucky $O(1)$ factors. If we want a superconformal flavor model that works at $\operatorname{small} \tan \beta$, we should additionally generate suppression factors for $\overline{\mathbf{5}}$ 's. The tradeoff between $\tan \beta$ and $\epsilon_{\bar{F}_{i}, \bar{H}}$ is expressed in eqs. (2.8) and (2.9).

Without modifying our theory, the lowest dimension coupling to a Standard Model $\overline{\mathbf{5}}$ is $\bar{Q}^{4} \bar{H}$ or $\bar{Q}^{4} \bar{F}_{i}$. However, a-maximization quickly rules this out as a possible marginal interaction, since $\bar{H}$ or $\bar{F}_{i}$ would be forced to have $R$-charge less than $2 / 3$, in violation of unitarity. An alternative approach is to add a pair of GUT singlets $S+S^{\prime}$ transforming as $(1, \square)+(1, \square)$ under $\mathrm{SU}(5)_{\text {GUT }} \times \mathrm{Sp}(2 \mathrm{~N}) .{ }^{6}$ The theory now admits couplings

$$
W_{\overline{\mathbf{5}}}=\bar{F}_{1} Q S+\bar{H} Q S^{\prime}
$$

\footnotetext{
${ }^{6}$ Note that the $\mathrm{Sp}(2 \mathrm{~N})$ global anomaly forces us to add fundamentals in pairs.
} 


\begin{tabular}{|c|c|c|c|c|c|c|c|c|c|}
\hline & $T_{i}$ & $\bar{F}_{i}$ & $H$ & $\bar{H}$ & $Q$ & $\bar{Q}$ & $S$ & $S^{\prime}$ & $A$ \\
\hline$F$ & 1 & $x$ & -2 & $-1-x$ & $1 / 2$ & $-1 / 2$ & $-x-1 / 2$ & $x+1 / 2$ & 0 \\
\hline
\end{tabular}

Table 13. Possible U(1) flavor charges that disallow low-dimension baryon- and lepton-number violating operators. The parameter $x$ is arbitrary.

To avoid problematic dimension 3 and 4 baryon- and lepton-number violating operators (like $H \bar{F}_{i}$ and $T_{i} \bar{F}_{j} \bar{F}_{k}$ ), we can impose any $\mathrm{U}(1)$ flavor symmetry in the family shown in table 13. This family is uniquely determined by the requirement of consistency with $W_{\mathbf{1 0}}$, the SM Yukawas, and either of the interactions $\bar{F}_{i} Q S$ or $\bar{H} Q S^{\prime}$. In particular, it's impossible to use a U(1) flavor symmetry to allow one of the interactions in $W_{\overline{5}}$ and disallow the other.

Without doing anything quantitative, we can anticipate that a model with marginal interactions $W_{\mathbf{1 0}}+W_{\overline{\mathbf{5}}}$ will give anomalous dimensions for $\bar{H}$ and $\bar{F}_{i}$ that are comparable to the anomalous dimension of $T_{1}$, since both come from three-field interactions with strongsector mesons. This will produce $\epsilon_{\bar{H}}$ and $\epsilon_{\bar{F}_{1}}$ factors that are unacceptably small if $W_{\overline{5}}$ remains marginal throughout the range $\mu \in\left[\Lambda_{c}, \Lambda\right]$. One possible resolution is that the pair $S+S^{\prime}$ develops a large mass and decouples above $\Lambda_{c}$, so that the anomalous dimensions $\gamma_{\bar{F}_{i}}$ and $\gamma_{\bar{H}}$ are exponentiated over a shorter running distance than $\gamma_{T_{1}}$. This then introduces the decoupling scale of $S, S^{\prime}$ as a new parameter into our theory, which diminishes the theory's predictivity.

However, one prediction that remains follows from the $\mathbb{Z}_{2}$ symmetry relating $\left(\bar{F}_{1}, S\right)$ and $\left(\bar{H}, S^{\prime}\right)$. We see that $R_{\bar{F}_{1}}=R_{\bar{H}}$, so $\epsilon_{\bar{F}_{1}}=\epsilon_{\bar{H}}$, regardless of the decoupling scale for $S, S^{\prime}$. In principle, if we could compute the $O(1)$ mixing angles that enter Yukawa couplings when exiting the conformal window (in particular, if we knew the origin of the violation of GUT mass relations), then this symmetry combined with eqs. (2.8) and (2.9) would yield a prediction for $\tan \beta$.

\section{A previously 'incalculable' model}

Several more examples of superconformal flavor models were presented in the initial paper on the subject [6]. For some of them, the $R$-charges could be determined uniquely from superpotential constraints and anomaly cancelation alone. However, such models typically involve a complicated superpotential with lots of marginal operators in order to fully constrain the space of trial $R$-charges. By contrast, models with simpler superpotentials were 'incalculable' at the time [6] was written, and the task of determining their viability as flavor models was left for future work. In this section, we will apply $a$-maximization to determine the $R$-charges for one such 'incalculable' model.

The model we consider has two exotic $\operatorname{Sp}(8)$ gauge groups, which we will refer to as $\operatorname{Sp}(8)$ and $\operatorname{Sp}(8)^{\prime}$. The matter content is given in table 14 . There are $N_{f}=9$ pairs of fundamentals under $\operatorname{Sp}(8)\left(N_{c}=4\right)$. In the absence of a superpotential, and ignoring the $\operatorname{Sp}(8)^{\prime}$ coupling, this gauge group enters a non-Abelian Coulomb phase because $3 / 2\left(N_{c}+\right.$ 1) $<N_{f}<3\left(N_{c}+1\right)$ [62]. In addition, there are $N_{f}^{\prime}=6$ pairs of fundamentals in a 


\begin{tabular}{|l|c|c|c|}
\hline & $\mathrm{SU}(5)_{\text {GUT }}$ & $\mathrm{Sp}(8)$ & $\mathrm{Sp}(8)^{\prime}$ \\
\hline$Q$ & $\overline{\mathbf{1 0}}$ & $\square$ & 1 \\
$L, M, J_{1 \ldots 6}$ & 1 & $\square$ & 1 \\
$\bar{Q}^{\prime}$ & $\mathbf{1 0}$ & 1 & $\square$ \\
$\bar{J}_{1,2}^{\prime}$ & 1 & 1 & $\square$ \\
\hline
\end{tabular}

Table 14. Matter content of the '10-centered' model presented in [6].

\begin{tabular}{|c|c|c|c|c|c|c|c|c|c|c|}
\hline$R_{T_{1}}$ & $R_{T_{2}}$ & $R_{Q}$ & $R_{L}$ & $R_{M}$ & $R_{J_{1}}$ & $R_{J_{2}}$ & $R_{J_{3}}$ & $R_{J_{4}}$ & $R_{J_{5}}$ & $R_{J_{6}}$ \\
\hline 1.104 & 1.107 & .401 & .494 & .491 & .502 & .498 & .501 & .499 & .500 & .500 \\
\hline
\end{tabular}

Table 15. $R$-charges in the '10-centered' model presented in [6].

confining $\operatorname{Sp}(8)^{\prime}$, which are introduced in order to allow all exotic states to decouple from the low-energy spectrum.

The theory is assumed to flow to a fixed point with the marginal interactions

$$
W_{\mathrm{int}}=\left(J_{1} J_{2}\right)^{2}+\left(J_{3} J_{4}\right)^{2}+\left(J_{5} J_{6}\right)^{2}+\left(L J_{1}\right)\left(J_{1} J_{3}\right)+T_{2} Q M+T_{1} Q L .
$$

While there is much that could be said about the structure of flows that could lead to this fixed point, as well as exit from the CFT regime and the decoupling of exotic states, here we will simply demonstrate that this model cannot be realistic due to the $R$-charges alone.

The superpotential and anomaly cancelation impose the constraints

$$
\begin{aligned}
& 2=R_{T_{1}}+R_{Q}+R_{L} \\
& 2=R_{T_{2}}+R_{Q}+R_{M} \\
& 2=2 R_{J_{1}}+2 R_{J_{2}} \\
& 2=2 R_{J_{3}}+2 R_{J_{4}} \\
& 2=2 R_{J_{5}}+2 R_{J_{6}} \\
& 2=2 R_{J_{1}}+R_{J_{3}}+R_{L} \\
& 0=2\left(N_{c}+1\right)+10\left(R_{Q}-1\right)+\left(R_{L}-1\right)+\left(R_{M}-1\right)+\sum_{i=1}^{6}\left(R_{J_{i}}-1\right) .
\end{aligned}
$$

Maximizing $a\left(R_{t}\right)$ subject to these constraints then yields the $R$-charges given in table 15 . From these results we can see immediately that generating a hierarchy is not possible due to the $R$-charges for $T_{1}$ and $T_{2}$ being approximately equal. Thus, we conclude that this model is not viable. ${ }^{7}$

\footnotetext{
${ }^{7}$ By contrast, the calculable examples in [6] do successfully generate a hierarchy over a small range of scales. However, we should note that they are still subject to the Landau pole constraint $\Lambda_{\mathrm{SU}(5)}<\Lambda$. The '10-centered model without proton decay' in [6] develops an SU(5) GUT (or SU(3)) Landau pole after $\sim 3.5$ decades of running, so cannot work down to $10 \mathrm{TeV}$, as the authors claim.
} 


\section{Conclusions}

It is not hard to imagine that the spectrum just below $M_{\mathrm{pl}}$ includes an exotic non-Abelian gauge group $G$ and vector-like matter charged under both $G$ and SU(5) GUT $_{\text {. Often such }}$ sectors are assumed to be lifted at a high scale and ignored for the sake of low-energy physics. But we've seen that some very simple exotic sectors can naturally generate flavor hierarchies in the Standard Model, dynamically distinguishing the different generations in a way determined simply by representation theory. For model builders working at the Planck scale, these kinds of exotic sectors are important to keep in mind as viable and well-motivated extensions to the MSSM.

Given this, it is worthwhile to investigate precisely which exotic sectors are phenomenologically interesting and viable. Luckily, $a$-maximization allows us to quantitatively evaluate a large class of superconformal flavor models that would be otherwise incalculable. Vector-like theories are a good starting point for model building because it's easy to ensure by adding mass terms that conformal symmetry is broken and all exotic states are lifted from the low-energy spectrum. However, we have seen that they are also highly constrained by demanding that the $\mathrm{SU}(5)_{\text {GUT }}$ coupling remain perturbative over the range of energies required to produce a reasonable Yukawa hierarchy. Roughly, a large hierarchy requires large anomalous dimensions, and therefore a strongly-coupled SCFT. But strong coupling usually requires a large exotic gauge group, and therefore forces us to include many GUT multiplets, driving the GUT $\beta$-function highly positive.

In this paper, we focused on possibly the simplest set of vector-like models: 10-centered models with a simple gauge group and a small number of superpotential operators. Among these, only models based on $\mathrm{Sp}(2 \mathrm{~N})$ with an anti-symmetric tensor seem to be efficient enough to easily evade the GUT Landau pole constraint. Some models based on SU(N) with an adjoint are also potentially viable. However, there are a few obvious generalizations that might yield other viable superconformal flavor models. Firstly, it would be interesting to do a systematic study of chiral exotic sectors. These might be better able to avoid the Landau pole constraint because large GUT multiplets needn't come in vector-like pairs. However, ensuring that all exotic states decouple is clearly a more delicate issue. Secondly, one might try finding models where a large number of composite operators in non-trivial GUT multiplets become free, in order to exploit the negative contribution to $\beta_{g_{5}}$ from $\{L, M\}$ pairs discussed in sections 2.3 and 3.3. Alternatively, one could investigate extending the exotic sector to include fields that don't couple directly to $T_{1}$ or $T_{2}$ in the superpotential, but affect their anomalous dimensions through other marginal superpotential couplings (as in [6]). Further, one might generalize to non-simple gauge groups, or include additional $\mathrm{U}(1)$ or discrete symmetries to constrain the allowed marginal operators.

We suspect that in each of these generalized classes of models, the Landau pole constraint allows only a few possibilities. Meanwhile, in non-GUT models, requiring that the $\mathrm{SU}(3)$ coupling remain perturbative should provide an even stronger constraint, since $\alpha_{\text {strong }}$ is strictly greater than $\alpha_{\mathrm{GUT}}$ and we lose the negative $\beta$-function contribution from $X Y$ gauge bosons. It seems unlikely that a flavor CFT could exist down to the $\mathrm{TeV}$ scale unless we are willing to give up on the idea of perturbative unification. 
Superconformal flavor models are related via AdS/CFT to 5D warped flavor models, which generate Yukawa hierarchies through sequestering in an extra dimension. In the dual picture to our setup, the Higgs, $T_{3}$, and other fields decoupled from the exotic sector are localized in the UV, ${ }^{8}$ while $T_{1}$ and $T_{2}$ correspond to massive fields localized near the IR. Warped flavor models are usually analyzed in the 5D supergravity limit, which corresponds to a large- $N$ limit in the dual CFT. However, large- $N$ CFTs are precisely those for which the Landau pole constraint is strongest, and one might worry that this places a severe restriction on calculable warped flavor models. Certainly, there seem to be only a limited number of viable superconformal flavor models even at small- $N$, and it would be interesting to better understand the nature of these limitations in a supergravity description.

It is also suggestive that viable superconformal flavor models can fit nicely below $M_{\mathrm{pl}}$, with conformal symmetry broken near $M_{\mathrm{GUT}}$. The exotic sectors we write down are similar to previously considered models of GUT breaking [63-65], and it would be interesting to try to combine the physics of conformal symmetry breaking and GUT breaking (and perhaps even SUSY breaking) in some way. To this end, it would be good to better understand the vacuum structure of the more realistic models in the presence of various relevant deformations. Further, after combining these flavor models with a more realistic picture of GUT physics and SUSY breaking, it would be interesting to see if deviations from GUT mass relations in the lighter generations can be accommodated at a more quantitative level.

Finally, we would like to stress that without a full understanding of the origin of the Yukawa hierarchies, one doesn't know the extent to which the 'SUSY flavor problem' is really a problem. In the present scenario, scalar mass operators which are potentially flavor-violating at a high scale will also receive suppressions by the CFT dynamics [32, 33]. It would be interesting, for example, to extend the analysis of $[35,36]$ on flavor constraints to the present case, where SUSY-breaking operators may receive extra suppressions due to having different anomalous dimensions under the strong dynamics. This may then help guide us to a more coherent picture of what low-scale measurements can tell us about the relationship between flavor dynamics and the mediation of supersymmetry breaking. If we are lucky, the mechanism underlying the flavor hierarchies will then leave its imprint at the LHC, and measurements of the superpartner spectrum will help us to come several steps closer to unraveling the deep mysteries of nature.

\section{Acknowledgments}

We thank Clay Cordova, Nathaniel Craig, Yanou Cui, Ken Intriligator, Andrey Katz, John Mason, David Morrissey, Lisa Randall, Matt Schwartz, and Brian Wecht for helpful comments and conversations. This work is supported in part by the Harvard Center for the Fundamental Laws of Nature and by NSF grant PHY-0556111. DP would like to thank the Galileo Galilei Institute for its hospitality during the completion of this work.

\footnotetext{
${ }^{8}$ Note that we are relying on SUSY to solve the hierarchy problem, so there is no need to put the Higgs in the IR.
} 


\section{A Sketch of a proof of $a$-maximization}

This appendix contains no new material, but rather is intended as a self-contained review of the proof of $a$-maximization, which originally appeared in [15], based on results from [66].

The claim that $R$ locally maximizes

$$
a\left(R_{t}\right)=\frac{3}{32}\left[3 \operatorname{Tr}\left(R_{t}^{3}\right)-\operatorname{Tr}\left(R_{t}\right)\right]
$$

where $R_{t}=R_{0}+\sum_{I} s_{I} F_{I}$ is equivalent to the statements

$$
\begin{aligned}
& \text { - } \frac{32}{3} \frac{\partial a}{\partial s_{I}}=9 \operatorname{Tr}\left(R R F_{I}\right)-\operatorname{Tr}\left(F_{I}\right)=0 \text { for each } F_{I} . \\
& \text { - } \frac{32}{3} \frac{\partial^{2} a}{\partial s_{I} \partial s_{J}}=18 \operatorname{Tr}\left(R F_{I} F_{J}\right) \quad \text { is a negative-definite matrix. }
\end{aligned}
$$

Their proofs are essentially independent, and we will show each in turn.

\section{A.1 Proof of eq. (A.2)}

eq. (A.2) is a relation between the mixed anomaly $\left\langle\partial^{\rho} J_{\rho}^{I} J_{\mu}^{R} J_{\nu}^{R}\right\rangle \propto \operatorname{Tr}\left(F_{I} R R\right)$ and the gravitational anomaly $\left\langle\partial^{\rho} J_{\rho}^{I} T_{\mu \sigma} T_{\nu \gamma}\right\rangle \propto \operatorname{Tr}\left(F_{I}\right)$. In a supersymmetric theory, the flavor currents $J_{I}^{\mu}$ live in vector superfields $J_{I}(z)$ with

$$
J_{I}^{\mu}(x)=\left.\sigma_{\alpha \dot{\alpha}}^{\mu}\left[\nabla^{\alpha}, \bar{\nabla}^{\dot{\alpha}}\right] J_{I}(z)\right|_{\theta=0},
$$

where $z$ is a superspace coordinate standing for $(x, \theta, \bar{\theta})$. Meanwhile the $R$-current $J_{\mu}^{R}$, the stress tensor $T_{\mu \nu}$, and the supersymmetry currents $\left\{S_{\mu}^{\alpha}, \bar{S}_{\mu \dot{\alpha}}\right\}$, are components of a single "supercurrent," [67]

$$
\begin{aligned}
T_{\mu}(z)= & J_{\mu}^{R}(x)+\theta^{\alpha}\left(S_{\mu \alpha}+\frac{1}{3}\left(\sigma_{\mu} \bar{\sigma}^{\rho} S_{\rho}\right)_{\alpha}\right)+\bar{\theta}_{\dot{\alpha}}\left(\bar{S}_{\mu}^{\dot{\alpha}}+\frac{1}{3} \epsilon^{\dot{\alpha} \dot{\beta}}\left(\bar{S}_{\rho} \bar{\sigma}^{\rho} \sigma_{\mu}\right)_{\dot{\beta}}\right) \\
& +\left(\theta \sigma^{\nu} \bar{\theta}\right)\left(2 T_{\mu \nu}-\frac{2}{3} \eta_{\mu \nu} T+\frac{1}{4} \epsilon_{\mu \nu \sigma \rho} \partial^{[\rho} J_{R}^{\sigma]}\right)+\ldots
\end{aligned}
$$

The supercurrent satisfies the conservation law

$$
\bar{\nabla}^{\dot{\alpha}} T_{\alpha \dot{\alpha}}=\nabla_{\alpha} L_{T}
$$

where $L_{T}$ (a chiral superfield) is the trace anomaly, equal to the variation of the action with respect to the chiral compensator in supergravity. In a conformal theory on a flat geometry with no background fields, $L_{T}$ vanishes.

Notice that both correlation functions $\left\langle J_{\rho}^{I} J_{\mu}^{R} J_{\nu}^{R}\right\rangle$ and $\left\langle J_{\rho}^{I} T_{\mu \sigma} T_{\nu \gamma}\right\rangle$ occur as components of the superfield correlator

$$
\left\langle T_{\mu}\left(z_{1}\right) T_{\nu}\left(z_{2}\right) J_{I}\left(z_{3}\right)\right\rangle .
$$

It follows that both anomalies $\operatorname{Tr}\left(R R F_{I}\right)$ and $\operatorname{Tr}\left(F_{I}\right)$ are determined by this correlator. $\left\langle T_{\mu} T_{\nu} J_{I}\right\rangle$ will generically have singularities as the points $z_{i}$ approach each other. This isn't 
a problem in an isolated CFT. However, if we try to couple the theory to background fields $\left\{E^{\mu}, A^{I}\right\}$ via a coupling $\delta \mathcal{L}=\int d^{4} \theta\left(E^{\mu} T_{\mu}+A^{I} J_{I}\right)$, then non-integrable singularities in correlators give rise to divergences in the perturbation expansion $\left\langle\exp \left(i \int d^{4} x \delta \mathcal{L}\right)\right\rangle$. To make sense of the theory in non-trivial backgrounds, we need to regularize the nonintegrable singularities. (For the asymptotically-free theories we consider in this paper, the regularization comes about physically from the fact that the CFT emerges from a different UV theory where the correlators are better-behaved at short distances.) Regularization introduces anomalous contact terms into the flavor-current conservation law,

$$
\bar{D}_{3}^{2}\left\langle T_{\mu}\left(z_{1}\right) T_{\nu}\left(z_{2}\right) J_{I}\left(z_{3}\right)\right\rangle=\text { contact terms. }
$$

And we can read off the anomaly coefficients $\operatorname{Tr}\left(R R F_{I}\right)$ and $\operatorname{Tr}\left(F_{I}\right)$ from different $\theta$ components of these contact terms.

So far, these considerations have been true in any supersymmetric theory. However the enlarged symmetry group of a SCFT imposes additional constraints. In particular, $J_{I}$ and $T_{\mu}$ become primary operators and their three-point function $\left\langle T_{\mu}\left(z_{2}\right) T_{\nu}\left(z_{3}\right) J_{I}\left(z_{1}\right)\right\rangle$ is uniquely determined up to an overall constant by superconformal symmetry [66]. This immediately implies that $\operatorname{Tr}\left(R R F_{I}\right)$ and $\operatorname{Tr}\left(F_{I}\right)$ are proportional to each other, with a constant that's universal for any SCFT. We can fix this constant by examining the special case of a free chiral superfield, which has $R$-charge $\frac{2}{3}$ and a single U(1) flavor symmetry $F$. In this case, $\operatorname{Tr}(R R F)=\operatorname{Tr}\left(\left(\frac{2}{3}-1\right)^{2} F\right)=\frac{1}{9} \operatorname{Tr}(F)$, which suffices to establish eq. (A.2).

\section{A.1.1 Determining $\left\langle T_{\mu} T_{\nu} J_{I}\right\rangle$ from superconformal invariance}

Since many readers may be unfamiliar with the results of [66], we'd like to go into greater detail about how superconformal symmetry fixes $\left\langle T_{\mu} T_{\nu} J_{I}\right\rangle$, and in turn the relation between $\operatorname{Tr}\left(R R F_{I}\right)$ and $\operatorname{Tr}\left(F_{I}\right) \cdot{ }^{9}$

Superconformal primary operators $\mathcal{O}$ are characterized by their spin, and weights $(q, \bar{q})$ such that $\operatorname{dim}(\mathcal{O})=q+\bar{q}$ and $R(\mathcal{O})=\frac{2}{3}(q-\bar{q})$. The supercurrent $T_{\mu}$ has $q=\bar{q}=\frac{3}{2}$, while the flavor currents $J_{I}$ have $q=\bar{q}=1$. These data completely determine an operator's transformation properties under superconformal transformations, along with all two-point functions (up to constants). In particular for the flavor currents, we have

$$
\left\langle J_{I}(z) J_{J}(0)\right\rangle=\frac{c_{I J}}{x_{+}^{2} x_{-}^{2}}
$$

where $x_{ \pm}$are chiral and anti-chiral coordinates $x_{ \pm}^{\mu}=x^{\mu} \pm i \theta \sigma^{\mu} \bar{\theta}$, and $c_{I J}$ are constants. Let us pick a basis such that $c_{I J}=\delta_{I J}$, and focus on a single flavor current $J_{I}=J$.

The superconformal group is generated by super-Poincare transformations and inversions which act on superspace as

$$
x_{\mp}^{\prime}=\frac{x_{ \pm}}{x_{ \pm}^{2}}, \quad \theta^{\prime}=-i \frac{\left(x_{-} \cdot \sigma\right) \bar{\theta}}{x_{-}^{2}}, \quad \bar{\theta}^{\prime}=i \frac{\theta\left(x_{+} \cdot \sigma\right)}{x_{+}^{2}} .
$$

\footnotetext{
${ }^{9}$ Though we will directly follow calculations in [66], we will not make use of some of the more specialized notation, in the interest of making this section as accessible as possible.
} 
Under an inversion $z \rightarrow z^{\prime}, J$ and $T_{\mu}$ transform as

$$
\begin{aligned}
J(z) & \rightarrow J^{\prime}(z)=\frac{1}{x_{+}^{2} x_{-}^{2}} J\left(z^{\prime}\right) \\
T_{\mu}(z) & \rightarrow T_{\mu}^{\prime}(z)=\frac{I_{\mu \nu}(z)}{\left(x_{+}^{2} x_{-}^{2}\right)^{3 / 2}} T^{\nu}\left(z^{\prime}\right),
\end{aligned}
$$

where

$$
I_{\mu \nu}(z)=\frac{\operatorname{tr}\left(\sigma_{\mu} \bar{\sigma}_{\rho} \sigma_{\nu} \bar{\sigma}_{\sigma}\right) x_{-}^{\rho} x_{+}^{\sigma}}{2\left(x_{-}^{2} x_{+}^{2}\right)^{1 / 2}} .
$$

As is perhaps familiar from the bosonic case, three-point functions can be constructed from two-point functions together with OPE relations. For the correlator (A.8), this works as follows. By (super)translation invariance, it suffices to find $\left\langle T_{\mu}\left(z_{1}\right) T_{\nu}\left(z_{2}\right) J(0)\right\rangle$. We will compute this as $\lim _{z_{3} \rightarrow 0}\left\langle T_{\mu}\left(z_{1}\right) T_{\nu}\left(z_{2}\right) J\left(z_{3}\right)\right\rangle$. Consider an inversion around 0 , taking $z_{i} \rightarrow z_{i}^{\prime}$ in the correlator

$$
\lim _{z_{3} \rightarrow 0}\left\langle T_{\mu}\left(z_{1}\right) T_{\nu}\left(z_{2}\right) J\left(z_{3}\right)\right\rangle=\lim _{z_{3} \rightarrow 0} \frac{I_{\mu}{ }^{\rho}\left(z_{1}\right) I_{\nu}{ }^{\sigma}\left(z_{2}\right)}{\left(x_{1+}^{2} x_{1-}^{2} x_{2+}^{2} x_{2-}^{2}\right)^{3 / 2}}\left\langle T_{\rho}\left(z_{1}^{\prime}\right) T_{\sigma}\left(z_{2}^{\prime}\right) J\left(z_{3}^{\prime}\right)\right\rangle \frac{1}{x_{3+}^{2} x_{3-}^{2}} .
$$

Now the point $z_{3}^{\prime}$ is approaching $\infty$ while $z_{1}^{\prime}$ and $z_{2}^{\prime}$ remain bounded, so we can safely use the TT operator product expansion,

$$
T_{\mu}\left(z_{1}^{\prime}\right) T_{\nu}\left(z_{2}^{\prime}\right) \sim \ldots+t_{\mu \nu}\left(z_{1}^{\prime}, z_{2}^{\prime}\right) J\left(z_{2}^{\prime}\right)+\ldots
$$

where "..." represents other operators. We'll see shortly that the $J\left(z_{2}^{\prime}\right)$ term above is the only one that can contribute to the correlator (A.8). Note for the moment that the only operators that have a nonvanishing two-point function with $J$ are $J$ and its descendants (obtained by acting on $J$ with momentum and supersymmetry generators).

Using the OPE (A.16), the right-hand side of (A.15) becomes

$$
\frac{I_{\mu}{ }^{\rho}\left(z_{1}\right) I_{\nu}{ }^{\sigma}\left(z_{2}\right)}{\left(x_{1+}^{2} x_{1-}^{2} x_{2+}^{2} x_{2-}^{2}\right)^{3 / 2}} t_{\rho \sigma}\left(z_{1}^{\prime}, z_{2}^{\prime}\right) \lim _{z_{3} \rightarrow 0}\left\langle J\left(z_{2}^{\prime}\right) J\left(z_{3}^{\prime}\right)\right\rangle \frac{1}{x_{3+}^{2} x_{3-}^{2}}+\ldots
$$

where "..." now represents the contribution from descendants of $J$. As $z_{3}^{\prime} \rightarrow \infty$, the factor $\left(x_{3+}^{2} x_{3-}^{2}\right)^{-1}$ grows like $x_{3}^{\prime 4}$, while the two-point function $\left\langle J\left(z_{2}^{\prime}\right) J\left(z_{3}^{\prime}\right)\right\rangle$ dies like $x_{3}^{\prime-4}$. By contrast, $\left\langle\mathcal{O}\left(z_{2}^{\prime}\right) J\left(z_{3}^{\prime}\right)\right\rangle$ dies faster than $x_{3}^{\prime-4}$ whenever $\mathcal{O}$ is a descendant of $J$. Hence, contributions from such operators don't survive in the limit $z_{3}^{\prime} \rightarrow \infty$. In fact, since shifting $J\left(z_{2}^{\prime}\right) \rightarrow J\left(z_{2}^{\prime}+\delta z\right)$ changes $J\left(z_{2}^{\prime}\right)$ by descendants, we can freely replace $J\left(z_{2}^{\prime}\right)$ with $J(0)$ in the limit, so the last two factors in (A.17) become

$$
\lim _{z_{3} \rightarrow 0}\left\langle J\left(z_{2}^{\prime}\right) J\left(z_{3}^{\prime}\right)\right\rangle \frac{1}{x_{3+}^{2} x_{3-}^{2}}=\lim _{z_{3} \rightarrow 0}\left\langle J(0) J\left(z_{3}^{\prime}\right)\right\rangle \frac{1}{x_{3+}^{2} x_{3-}^{2}}=1 .
$$

This leaves us with

$$
\left\langle T_{\mu}\left(z_{1}\right) T_{\nu}\left(z_{2}\right) J(0)\right\rangle=\frac{I_{\mu}{ }^{\rho}\left(z_{1}\right) I_{\nu}{ }^{\sigma}\left(z_{2}\right)}{\left(x_{1+}^{2} x_{1-}^{2} x_{2+}^{2} x_{2-}^{2}\right)^{3 / 2}} t_{\rho \sigma}\left(z_{1}^{\prime}, z_{2}^{\prime}\right) .
$$


Finally, we will show that the OPE coefficient $t_{\mu \nu}$ is determined up to a constant by symmetry and conservation of the supercurrent. By supertranslation invariance, we may compute $t_{\mu \nu}(z) \equiv t_{\mu \nu}(z, 0)$. Exchanging $T_{\mu} \leftrightarrow T_{\nu}$ gives $t_{\mu \nu}(z)=t_{\nu \mu}(-z)$. Also, $t_{\mu \nu}$ is real and thus a function of $x^{\mu}$ and $p^{\mu} \equiv \theta \sigma^{\mu} \bar{\theta}$ alone. Since $p_{\mu} p_{\nu}=\frac{1}{4} \eta_{\mu \nu} p^{2}$ and $p^{2} p_{\mu}=0$, we see that the most general form of $t_{\mu \nu}$ with the right scaling dimension and symmetry properties is

$$
t_{\mu \nu}(z)=\frac{\eta_{\mu \nu}}{x^{4}}\left(A+B \frac{p^{2}}{x^{2}}\right)+\frac{x_{\mu} x_{\nu}}{x^{6}}\left(C+D \frac{p^{2}}{x^{2}}\right)+\frac{E}{x^{6}} \epsilon_{\mu \nu \rho \sigma} x^{\rho} p^{\sigma},
$$

where $A, B, C, D, E$ are real constants.

The conservation law eq. (A.7) implies $\bar{D}^{\dot{\alpha}} t_{\alpha \dot{\alpha} \beta \dot{\beta}}=0$, or equivalently

$$
\theta \sigma^{\rho} \bar{\sigma}^{\mu}\left(\frac{\partial}{\partial p^{\rho}}-i \frac{\partial}{\partial x^{\rho}}\right) t_{\mu \nu}=0 .
$$

Inserting the general form (A.20) gives $C=-4 A$ and $B=D=E=0$, which completes the proof that $\left\langle T_{\mu} T_{\nu} J\right\rangle$ is determined up to an overall constant.

\section{A.2 Proof of eq. (A.3)}

The trace $\operatorname{Tr}\left(R F_{I} F_{J}\right)$ is proportional to the $R$-current anomaly $\partial_{\mu} J_{R}^{\mu}$ in the presence of background $\mathrm{U}(1)$ flavor fields $A_{I}$. This is encoded in the supertrace $L_{T} \sim \operatorname{Tr}\left(W_{I}^{\alpha} W_{J \alpha}\right)$. However, $L_{T}$ also encodes the trace of the stress-energy tensor $\Theta=T_{\mu}^{\mu}[68]$,

$$
\begin{aligned}
i \nabla_{\mu} J_{R}^{\mu} & =\left.\frac{1}{2}\left\{\nabla^{\alpha}, \bar{\nabla}^{\dot{\alpha}}\right\} T_{\alpha \dot{\alpha}}\right|_{\theta=0}=\left.\left(\nabla^{2} L_{T}-\bar{\nabla}^{2} \bar{L}_{T}\right)\right|_{\theta=0} \\
\Theta & =\left.\frac{3}{8}\left[\nabla^{\alpha}, \bar{\nabla}^{\dot{\alpha}}\right] T_{\alpha \dot{\alpha}}\right|_{\theta=0}=\left.\frac{3}{4}\left(\nabla^{2} L_{T}+\bar{\nabla}^{2} \bar{L}_{T}\right)\right|_{\theta=0} .
\end{aligned}
$$

These relations fix $\Theta(x)$ in terms of the $R$-anomaly, a familiar fact that we used, for instance to calculate the $\mathrm{SU}(5)_{\mathrm{GUT}} \beta$-function in eq. (3.3). Letting $\langle\cdot\rangle_{A}$ denote an expectation value in the flavor background, we have

$$
\begin{aligned}
\left\langle\partial_{\mu} J_{R}^{\mu}(x)\right\rangle_{A} & =\operatorname{Tr}\left(R F_{I} F_{J}\right) \frac{1}{16 \pi^{2}} F_{I}^{\mu \nu} \widetilde{F}_{J \mu \nu}(x) \\
\langle\Theta(x)\rangle_{A} & =-\operatorname{Tr}\left(R F_{I} F_{J}\right) \frac{3}{32 \pi^{2}} F_{I}^{\mu \nu} F_{J \mu \nu}(x) .
\end{aligned}
$$

So we can compute $\operatorname{Tr}\left(R F_{I} F_{J}\right)$ by examining how the $A_{I}$ break scale invariance, leading to nonzero $\Theta$.

Conformal symmetry completely determines the two-point function of flavor currents

$$
\left\langle J_{I}^{\mu}(x) J_{J}^{\nu}(0)\right\rangle=\frac{\tau_{I J}}{(2 \pi)^{4}}\left(\square g^{\mu \nu}-\partial^{\mu} \partial^{\nu}\right) \frac{1}{x^{4}}
$$

where $\tau_{I J}$ is a constant matrix which must be positive-definite in a unitary theory $\left(\tau_{I J}\right.$ is related to $c_{I J}$ in eq. (A.10) by $c_{I J}=\frac{\tau_{I J}}{(4 \pi)^{4}}$ ). This has a non-integrable singularity at $x=0$, which we must regulate in the presence of the flavor fields $A_{I}^{\mu}$. If we introduce a cutoff at the scale $\frac{1}{x} \sim \Lambda$, we need the counterterm

$$
\delta \mathcal{L}=\frac{\tau_{I J}}{32 \pi^{2}} \log \frac{\mu}{\Lambda} F_{I}^{\mu \nu} F_{J \mu \nu}
$$


to keep the physics fixed. Thus we have

$$
\left\langle\int d^{4} x \Theta(x)\right\rangle_{A}=\mu \frac{d}{d \mu}\langle 1\rangle_{A}=\left\langle\int d^{4} x \frac{\tau_{I J}}{32 \pi^{2}} F_{I}^{\mu \nu} F_{J \mu \nu}(x)\right\rangle_{A},
$$

which implies $\operatorname{Tr}\left(R F_{I} F_{J}\right)=-\frac{1}{3} \tau_{I J}$. Recall that $\tau_{I J}$ is positive-definite by unitarity, so we conclude that $\operatorname{Tr}\left(R F_{I} F_{J}\right)$ is negative-definite.

\section{B CFT exit and dual descriptions}

What happens when conformal symmetry is broken? In this appendix we will consider more carefully what happens when we deform our vector-like models with mass terms. In general we will find that this leads to non-trivial mass mixing between the fields $T_{i}$ and composite states in the CFT, such that the Yukawa interactions among light modes are modified by mixing angles. The precise mixing angles depend non-trivially on the mass deformations. We will not be able to compute them exactly, but we can say something about their structure in certain limits. Along the way, we'll learn something about Seiberg duality in superconformal flavor models.

We will carry out much of the discussion in the general context of $\mathrm{SU}\left(\mathrm{N}_{\mathrm{c}}\right)$ supersymmetric QCD with $N_{f}$ flavors $\{Q, \bar{Q}\}$ coupled to singlets $T$, specializing to the toy model of section 2.2 (where $Q=\{S, X\}$ ) when appropriate. In order to determine the mixing angles mentioned above, we will need to know the masses of fluctuations around the vacua of our theory at the scale where conformal symmetry is broken. In some cases our theory (considering just one generation for simplicity) can be described an effective superpotential

$$
W=m_{1} T_{1} \Phi_{1}+m_{2} \Phi_{2} \Phi_{1}
$$

where $\left\{T_{1}, \Phi_{1}, \Phi_{2}\right\}$ are canonically normalized fields. Letting $\sin \alpha=m_{2} / \sqrt{m_{1}^{2}+m_{2}^{2}}$, we see that the linear combination $T_{1} \cos \alpha+\Phi_{2} \sin \alpha$ becomes massive with $\Phi_{1}$, leaving a canonically normalized massless mode $T^{\prime}=T_{1} \sin \alpha-\Phi_{2} \cos \alpha$ with the Yukawa interactions

$$
W=\sin ^{2} \alpha \lambda_{11}^{u} T^{\prime} T^{\prime} H+\sin \alpha \lambda_{1 j}^{u} T^{\prime} T_{j} H+\sin \alpha \lambda_{1 j}^{d} T^{\prime} \bar{F}_{j} \bar{H} .
$$

If the mixing angle is $O(1)$, this will not be an important effect given that we have already assumed arbitrary $O(1)$ couplings in the UV. On the other hand, if $\alpha$ is small then this mixing will behave like an additional contribution to $\epsilon_{T_{1}}$. In the main body of this text we have implicitly assumed the former rather than the latter. Here we will try to explore the extent to which this assumption is justified.

In order to estimate these mixing effects, there are then two important questions we should try to answer. Firstly, what are the appropriate degrees of freedom to describe fluctuations in the exotic sector that couple to $T_{1}$ at or below the scale of conformal symmetry breaking? Secondly, what is the Kähler potential for those degrees of freedom? The answer to the first question depends on how strongly coupled the CFT is. When $N_{f}$ is near $3 N_{c}$, we should use the electric description in terms of elementary quarks $\{Q, \bar{Q}\}$. At the other end of the conformal window where $N_{f}$ is near $\frac{3}{2} N_{c}$, the magnetic variables $\{M, q, \bar{q}\}$ are more appropriate. 
As a first approximation, we will take the Kähler potential to be determined only by the anomalous dimensions in the CFT, right down to the scale of conformal symmetry breaking $\Lambda_{c}$. That is, we will ignore the contribution of mass deformations to the anomalous dimensions. These effects are not calculable in a strongly-coupled theory, but it is reasonable to make the assumption that these "threshold effects" are at most $O(1)$. We will also for the most part neglect RGE running below $\Lambda_{c}$. For instance, if conformal symmetry is broken when some of the electric quarks get mass $\Lambda_{c}$ while others remain relatively light, we will eschew the details of running and matching between $\Lambda_{c}$ and the lighter scale. Ignoring these effects is justifiable if the theory is weakly coupled below the scale $\Lambda_{c}$ and there are no large logarithms, or if the remaining fields are simply decoupled from the visible sector.

At a scale $\mu$, the electric theory has an effective superpotential and gauge kinetic terms given by

$$
W_{\text {electric }}=h_{*} T Q \bar{Q}+\operatorname{Tr}(m(\mu) Q \bar{Q})+\frac{b}{16 \pi^{2}} \log \left(\frac{\mu}{\Lambda_{h}}\right) \operatorname{Tr}\left(W^{\alpha} W_{\alpha}\right),
$$

where $T, Q$, and $\bar{Q}$ are canonically normalized and we suppress flavor indices. The coupling $h_{*}$ is at its fixed-point value, while the mass matrix is given by

$$
m(\mu)=m_{U V}\left(\frac{\mu}{\Lambda}\right)^{\frac{3}{2} R(Q \bar{Q})-2} .
$$

Finally, $\Lambda_{h}$ is the holomorphic scale ${ }^{10}$

$$
\Lambda_{h}=\mu \exp \left(\frac{2 \pi i \tau_{*}}{b}\right)=\mu \exp \left(-\frac{8 \pi^{2}}{b g_{*}^{2}}+i \frac{\theta}{b}\right)
$$

where $b=3 N_{c}-N_{f}$ and $g_{*}$ is the fixed-point value of the gauge coupling.

If our CFT is weakly coupled $\left(N_{c} g_{*}^{2} / 8 \pi^{2} \ll 1\right)$, then the electric quarks $Q, \bar{Q}$ are good degrees of freedom. The mass term becomes important when $m(\mu) \sim \mu$, at which point $Q$ and $\bar{Q}$ are lifted from the spectrum. It's clear that perturbatively there is no mass mixing between $T$ and exotic states, and the primary effect of integrating out the quarks at $\mu=\Lambda_{c}$ is to correct the Kähler potential

$$
T^{\dagger} T \rightarrow T^{\dagger} T\left(1+\frac{c\left|h_{*}\right|^{2}}{16 \pi^{2}}+\ldots\right)
$$

and introduce higher-dimension operators suppressed by $\Lambda_{c}$.

By contrast, when the electric theory is very strongly coupled, a more appropriate description of the degrees of freedom is in terms of the dual magnetic variables $\{M, q, \bar{q}\}$

\footnotetext{
${ }^{10}$ It may seem unusual that a CFT has a distinguished scale. However, this is just an artifact of our choice of normalization for the quarks, and doesn't reflect breaking of conformal invariance. $\Lambda_{h}$ is the position of the ostensible Landau pole if the gauge coupling were to undergo naïve one-loop running from the scale $\mu$. But the anomalous dimensions of the quarks modify the running of $g$, and ensure that this pole is unphysical. If we flow to a different energy scale $\mu^{\prime}$, we should rescale the quarks $Q, \bar{Q}$ to canonically normalize them. However, the rescaling anomaly then shifts $\Lambda_{h} \rightarrow \Lambda_{h}^{\prime}=\frac{\mu^{\prime}}{\mu} \Lambda_{h}$.
} 
with gauge group $\mathrm{SU}(\widetilde{\mathrm{N}})=\mathrm{SU}\left(\mathrm{N}_{\mathrm{f}}-\mathrm{N}_{\mathrm{c}}\right)$ [38]. When $T, M, q$, and $\bar{q}$ are canonically normalized at the scale $\mu$, the magnetic theory has an effective superpotential and gauge kinetic terms given by

$$
W_{\text {magnetic }}=\widetilde{h}_{*} \mu T M+\lambda_{*} M q \bar{q}+\mu \operatorname{Tr}(\widetilde{m}(\mu) M)+\frac{\widetilde{b}}{16 \pi^{2}} \log \left(\frac{\mu}{\widetilde{\Lambda}_{h}}\right) \operatorname{Tr}\left(W^{\alpha} W_{\alpha}\right),
$$

where $\widetilde{h}_{*}$ and the block matrix $\lambda_{*}$ are at their fixed-point values,

$$
\widetilde{\Lambda}_{h}=\mu \exp \left(\frac{2 \pi i \widetilde{\tau}_{*}}{\widetilde{b}}\right)=\mu \exp \left(-\frac{8 \pi^{2}}{\widetilde{b} \widetilde{g}_{*}^{2}}+i \frac{\widetilde{\theta}}{\widetilde{b}}\right)
$$

is the magnetic holomorphic scale, and $\widetilde{b}=3 \widetilde{N}-N_{f}$. Since the coupling between $T$ and $M$ is simply a mass term in this description, one approach would be to simply integrate out these massive fields. However, expanding the theory around its supersymmetric vacua will in general induce additional mass terms for the mesons $M$, leading to the mixing effects discussed above. In order to better understand these mixing effects we will for now leave $T$ in the description of the theory until we know which linear combination of fields becomes massive.

A partial dictionary between electric and magnetic variables follows from matching vacuum superpotentials. We will here only consider singlet couplings that lead to vacua where the VEV of $T$ (as well as the meson it couples to) vanishes, since otherwise the GUT group would be broken in a problematic way. We will also assume that $m(\mu)$ is of maximal rank. Since we only care about holomorphic information for the moment, we can be brazen about integrating out degrees of freedom without worrying about their physical masses.

In the electric theory, integrating out the quarks leaves pure $\mathrm{SU}\left(\mathrm{N}_{\mathrm{c}}\right) \mathrm{SYM}$ with scale $\Lambda_{L}=\left(\operatorname{det} m(\mu) \Lambda_{h}^{b}\right)^{1 / 3 N_{c}}$, which has $N_{c}$ vacua with superpotential

$$
W_{v a c}=N_{c} \Lambda_{L}^{3}=N_{c}\left(\operatorname{det} m(\mu) \Lambda_{h}^{b}\right)^{1 / N_{c}} .
$$

Meanwhile in the magnetic theory, the dual quarks have a meson-dependent mass matrix $\lambda_{*} M$. Integrating them out gives pure $\mathrm{SU}(\widetilde{\mathrm{N}}) \mathrm{SYM}$ with scale $\widetilde{\Lambda}_{L}=\left(\operatorname{det}\left(\lambda_{*} M\right) \widetilde{\Lambda}_{h}^{\widetilde{b}}\right)^{1 / 3 \widetilde{N}}$, which confines and leaves us with an effective superpotential

$$
W_{\text {eff }}(M)=\widetilde{h}_{*} \mu T M+\mu \operatorname{Tr}(\widetilde{m}(\mu) M)+\widetilde{N}\left(\operatorname{det}\left(\lambda_{*} M\right) \widetilde{\Lambda}_{h}^{\tilde{b}}\right)^{1 / \widetilde{N}} .
$$

Finally, extremizing $W_{\text {eff }}$, we find

$$
\begin{aligned}
\langle M\rangle & =\frac{1}{\mu}\left(\frac{(-1)^{\widetilde{N}} \operatorname{det}\left(\mu \widetilde{m}(\mu) \lambda_{*}^{-1}\right)}{\widetilde{\Lambda}_{h}^{\tilde{b}}}\right)^{1 / N_{c}} \widetilde{m}(\mu)^{-1} \\
W_{\mathrm{eff}}(\langle M\rangle) & =N_{c}\left(\frac{(-1)^{\widetilde{N}} \operatorname{det}\left(\mu \widetilde{m}(\mu) \lambda_{*}^{-1}\right)}{\widetilde{\Lambda_{h}^{b}}}\right)^{1 / N_{c}} .
\end{aligned}
$$

Matching $W_{\text {eff }}(\langle M\rangle)=W_{\text {vac }}$ then implies the relation

$$
\widetilde{m}(\mu)=m(\mu) \frac{\lambda_{*} \widehat{\Lambda}}{\mu},
$$

where $\widehat{\Lambda}^{N_{f}}=(-1)^{\widetilde{N}} \Lambda_{h}^{b} \widetilde{\Lambda}_{h}^{\widetilde{b}}=\mu^{N_{f}} e^{2 \pi i\left(\tau_{*}+\widetilde{\tau}_{*}+\widetilde{N} / 2\right)}$ 
Holomorphy only tells us about expectation values of chiral operators. If we're interested in physical degrees of freedom and masses, the correct picture of the magnetic vacua depends more intricately on the structure of the mass matrix $\widetilde{m}(\mu)$. For example, let us start by supposing that $\widetilde{m}(\mu)$ has a single large eigenvalue $\widetilde{m}_{1}$ at the scale $\mu=\Lambda_{c}$, where $\Lambda_{c} \sim \widetilde{m}_{1}$ is the scale at which the CFT description breaks down. Ignoring the other eigenvalues for the moment, the equation of motion $\lambda_{*} q_{1} \bar{q}_{1}=\Lambda_{c} \widetilde{m}_{1}$ implies that $q_{1}$ and $\bar{q}_{1}$ get VEVs, Higgsing the dual gauge group $\mathrm{SU}(\widetilde{\mathrm{N}}) \rightarrow \mathrm{SU}(\widetilde{\mathrm{N}}-1) .{ }^{11}$ At the same time, the VEVs $\left\langle q_{1}\right\rangle,\left\langle\bar{q}_{1}\right\rangle$ lift some of the meson flavors via mass terms

$$
W=\lambda_{*}\left\langle q_{1}\right\rangle \bar{q}_{i} M_{1 i}+\lambda_{*}\left\langle\bar{q}_{1}\right\rangle q_{i} M_{i 1}+\ldots
$$

Of course, the remaining meson flavors could remain somewhat light, but this might not be important for us.

Now, suppose in our toy model we take $\widetilde{m}_{S} \gg \widetilde{m}_{X}$. Then the GUT singlet dual quarks $\{s, \bar{s}\}$ get VEVs $\sim \sqrt{\Lambda_{c} \widetilde{m}_{S} / \lambda_{*}}$, yielding an effective superpotential

$$
W=\widetilde{h}_{*} \Lambda_{c} T_{1} M_{S \bar{X}}+\sqrt{\lambda_{*} \Lambda_{c} \widetilde{m}_{S}} \bar{x} M_{S \bar{X}}+\ldots,
$$

where $\bar{x}$ is a dual quark in the $\mathbf{1 0}$ representation of $\mathrm{SU}(5)_{\mathrm{GUT}}$. We see here that $T_{1}$ mixes with $\bar{x}$, and the mixing angle $\sin \alpha$ depends on the ratio of $\sqrt{\lambda}_{*}$ and $\widetilde{h}_{*}$, as well as the precise relation between $\Lambda_{c}$ and $\widetilde{m}_{1}$ and any threshold corrections in the Kähler potential.

Next let us consider the different limit in which all the masses are the same at $\mu=\Lambda_{c}$, i.e. $\widetilde{m}_{i j}\left(\Lambda_{c}\right)=\widetilde{m} \delta_{i j}$. In the limit where the magnetic theory is weakly-coupled, we can verify that the dual quarks are much heavier than the mesons, and should be integrated out. In this case the dual quark masses set the scale of conformal symmetry breaking, $\Lambda_{c} \sim \lambda_{*}\langle M\rangle$. Solving this equation for $\widetilde{m}$ then yields

$$
\widetilde{m} \sim-\lambda_{*}\left(\Lambda_{c}^{N_{c}-\widetilde{N} \widetilde{\Lambda}} \widetilde{b}_{h}^{1 / \widetilde{N}} \sim-\lambda_{*} \Lambda_{c} e^{2 \pi i \widetilde{\tau}_{*} / \widetilde{N}} \ll \Lambda_{c}\right.
$$

where the last relation holds (up to the phase) when $\widetilde{g}_{*}^{2} \widetilde{N} / 8 \pi^{2} \ll 1$ due to the exponential suppression. Further, we may estimate

$$
\left.\frac{\partial^{2} W_{\mathrm{eff}}}{\partial M^{2}}\right|_{M=\langle M\rangle} \sim \frac{\widetilde{m} \mu}{\langle M\rangle} \sim \lambda_{*} \widetilde{m} \ll \Lambda_{c},
$$

showing it is indeed physically sensible in this limit to integrate out $q, \bar{q}$, leaving us with an effective theory of mesons with superpotential $W_{\text {eff }}(M)$.

Specializing to our toy model, the superpotential after conformal symmetry breaking is

$$
W_{\mathrm{eff}}(\langle M\rangle+\widehat{M})=\widetilde{h}_{*} \Lambda_{c} T_{1} \widehat{M}_{S \bar{X}}+\lambda_{*} \widetilde{m} \widehat{M}_{X \bar{S}} \widehat{M}_{S \bar{X}}+\ldots,
$$

\footnotetext{
${ }^{11}$ This type of analysis is only trustworthy when $\widetilde{m}(\mu)$ has at most $N_{c}-1$ large eigenvalues. For a general rank- $N_{f}$ mass matrix, the equation of motion $\lambda_{*} q \bar{q}=\Lambda_{c} \widetilde{m}$ cannot be satisfied classically, since $\operatorname{rank}(q \bar{q}) \leq N_{c}$. This "rank condition" is violated non-perturbatively in the supersymmetric vacua, so there is no contradiction with eq. (B.11). In free magnetic theories, the rank condition implies the existence of metastable SUSY-breaking vacua [69]. In this paper, we will only consider supersymmetric vacua. However, it would be interesting to think about metastable SUSY breaking in superconformal flavor models, perhaps along the lines of [70].
} 
where $\widehat{M}$ are fluctuations around the $\operatorname{VEV}\langle M\rangle$. Note that integrating out $q, \bar{q}$ will give a correction to the Kähler potential of $\widehat{M}$ similar to eq. (B.6). However, in the weaklycoupled magnetic limit, $\widehat{M}$ is close to canonically normalized at $\mu=\Lambda_{c}$. This superpotential is then of the general form (B.1), so we find mass mixing between $T_{1}$ and $\widehat{M}_{X \bar{S}}$, with an angle depending on the fixed-point values of the couplings $\widetilde{h}_{*}, \lambda_{*}$, and $\widetilde{\tau}_{*}$. In the very weakly-coupled limit, $\widetilde{m} / \Lambda_{c}$ is suppressed as $e^{-8 \pi^{2} / \widetilde{N} \widetilde{g}_{*}^{2}}$ and hence we expect the mixing angle $\sin \alpha \ll 1$. On the other hand, it is important to note that approaching this weaklycoupled (Banks-Zaks) limit [71, 72] requires being at very large $N$, and none of the models considered in the present work are at sufficiently large $N$ so as to really approach this limit.

Finally, we would like to comment on the alternative approach of simply integrating out the $\{T, M\}$ pair at the top of the conformal window. Doing so and running down to arbitrary $\mu$ generates operators such as

$$
W=-\frac{\lambda_{*}}{\widetilde{h}_{*} \mu}\left(\frac{\mu}{\Lambda}\right)^{\frac{3}{2} R(q \bar{q})-1} y_{1 j}^{d}(q \bar{q}) \bar{F}_{j} \bar{H}+\ldots
$$

along with similar operators corresponding to the other Yukawa interactions. Note that these operators are generated at the scale $\Lambda$ with the naïve $1 / \Lambda$ suppression, but are then anomalously affected by the CFT dynamics at lower energies. In the case that the singlet dual quark $s$ acquires a VEV, these couplings lead to the same predictions as before after identifying the dual quark $\bar{x}$ with the low-energy 10. On the other hand, when the mesons acquire VEVs and the non-perturbative superpotential is important, the situation is slightly more subtle. In this case, the dual quark mass matrix is now a linear combination of $M$ and $\bar{F}_{j} \bar{H}$, and this linear combination will enter the non-perturbative superpotential. After expanding the non-perturbative superpotential around $\langle M\rangle$, the Yukawa couplings are then recovered by identifying $M_{X \bar{S}}$ with the low-energy 10. Similar considerations will apply to the $y_{1 j}^{u}$ couplings for $j \neq 1$. Somewhat more care is required to understand how the operator $y_{11}^{u}(q \bar{q})(q \bar{q}) H$ affects the non-perturbative superpotential when the dual quarks are integrated out. However, this could for example be obtained by using chiral ring relations to determine an effective glueball superpotential, which when minimized and expanded around $\langle M\rangle$ should recover the same predictions as before.

To summarize, here we've identified at least three kinds of behavior when conformal symmetry is broken, depending on how strongly-coupled the conformal sector is and the structure of the mass matrix $m(\mu)$ (or equivalently $\widetilde{m}(\mu)$ ). When the electric theory is weakly-coupled, exotic states are lifted and the Kähler potential of $T_{1}$ receives small corrections. When the magnetic theory is weakly coupled, $T_{1}$ can mix with either meson fluctuations or dual quarks, with different mixing angles depending on the structure of $\widetilde{m}(\mu)$. We expect a variety of mixing angles are possible away from these special cases. In this paper, we have assumed that RGE running gives the dominant contribution to the Yukawa hierarchies. This simplifies our analysis, but it is not guaranteed to be what nature chooses. Some models that we rule out as pure superconformal flavor models may be salvageable if the Yukawa hierarchies come from a hybrid of RGE running and mixing angles. 


\section{Models with two 3 -field or 4 -field $T_{i}$ couplings}

In this appendix, we list all vector-like exotic sectors with a simple, non-IR free gauge group and two representation-theoretically distinct three-field or four-field couplings to $T_{i}$ 's. In scanning over models, we have been aided by [73]. In many cases, these exotic sectors can be extended by adding GUT singlets, for instance to get more couplings to SM $\overline{5}$ 's. However, adding more matter makes the CFT less strongly coupled, and typically reduces the anomalous dimensions of $T_{1}$ and $T_{2}$. Regardless, here we only display the fields directly involved in giving anomalous dimensions to $T_{1}$ and $T_{2}$.

The condition that the two couplings $T_{1} \mathcal{O}_{1}$ and $T_{2} \mathcal{O}_{2}$ be representation-theoretically distinct is an elegant way to ensure that no symmetry forces $\gamma_{T_{1}}=\gamma_{T_{2}}$. Another strategry is to allow the two couplings to be representation-theoretically identical, but break the symmetry between them with other superpotential deformations. This is the solution used, for instance, in the model of section 4 (originally from [6]). However, we won't attempt to classify such models here.

In addition to the representation content of a superconformal flavor model, one has to decide which relevant deformations to include in the superpotential. We have not attempted to study all possible deformations here. Rather, we chose to focus in the main text on the phenomenologically interesting deformations of the most promising models.

\section{C.1 Models without 10's}

\begin{tabular}{|l|c|c|}
\hline & $\mathrm{SU}(5)_{\mathrm{GUT}}$ & $\mathrm{SU}(\mathrm{N})$ \\
\hline$Q_{1}+\bar{Q}_{2}$ & $\mathbf{5}+\overline{\mathbf{5}}$ & $\square$ \\
$\bar{Q}_{1}+Q_{2}$ & $\overline{\mathbf{5}}+\mathbf{5}$ & $\square$ \\
$A$ & $\mathbf{1}$ & Ad. \\
\hline
\end{tabular}

Table 16. Couplings $T_{1} \bar{Q}_{1} \bar{Q}_{2}+T_{2} \bar{Q}_{1} A \bar{Q}_{2}$.

\begin{tabular}{|l|c|c|}
\hline & $\mathrm{SU}(5)_{\text {GUT }}$ & $\mathrm{SO}(\mathrm{N})$ \\
\hline$Q_{1,2}+\bar{Q}_{1,2}$ & $2 \times(\mathbf{5}+\overline{\mathbf{5}})$ & $\square$ \\
$A$ & 1 & 日or $\square$ \\
\hline
\end{tabular}

Table 17. Couplings $T_{1} \bar{Q}_{1} \bar{Q}_{2}+T_{2} \bar{Q}_{1} A \bar{Q}_{2}$.

\begin{tabular}{|l|c|c|}
\hline & $\mathrm{SU}(5)_{\mathrm{GUT}}$ & $\mathrm{Sp}(2 \mathrm{~N})$ \\
\hline$Q+\bar{Q}$ & $\mathbf{5}+\overline{\mathbf{5}}$ & $\square$ \\
$A$ & $\mathbf{1}$ & $\boxminus$ \\
\hline
\end{tabular}

Table 18. Couplings $T_{1} \bar{Q} \bar{Q}+T_{2} \bar{Q} A \bar{Q}$. 


\begin{tabular}{|c|c|c|}
\hline & $\mathrm{SU}(5)_{\text {GUT }}$ & $\mathrm{SU}(3)$ \\
\hline$Q+S$ & $\mathbf{5}+\mathbf{1}$ & $\square$ \\
$\bar{Q}+\bar{S}$ & $\overline{\mathbf{5}}+\mathbf{1}$ & $\square$ \\
\hline
\end{tabular}

Table 19. Couplings $T_{1} Q^{3}+T_{2} \bar{S} \bar{Q}^{2}$

\begin{tabular}{|c|c|c|}
\hline & $\mathrm{SU}(5)_{\mathrm{GUT}}$ & $G_{2}$ \\
\hline$Q+\bar{Q}+S$ & $\mathbf{5}+\overline{\mathbf{5}}+\mathbf{1}$ & $\square$ \\
\hline
\end{tabular}

Table 20. Couplings $T_{1} Q^{3}+T_{2} S \bar{Q}^{2}$.

\section{C.2 Models with 10's}

\begin{tabular}{|c|c|c|}
\hline & $\mathrm{SU}(5)_{\text {GUT }}$ & $\mathrm{SU}(\mathrm{N})$ \\
\hline$X+\bar{Q}+S$ & $\mathbf{1 0}+\overline{\mathbf{5}}+\mathbf{1}$ & $\square$ \\
$\bar{X}+Q+\bar{S}$ & $\overline{\mathbf{1 0}}+\mathbf{5}+\mathbf{1}$ & $\bar{\square}$ \\
\hline
\end{tabular}

Table 21. Couplings $T_{1} \bar{X} S+T_{2} X Q$.

\begin{tabular}{|c|c|c|}
\hline & $\mathrm{SU}(5)_{\mathrm{GUT}}$ & $\mathrm{SU}(\mathrm{N})$ \\
\hline$X+Q_{1}+\bar{Q}_{2}$ & $\mathbf{1 0}+\mathbf{5}+\overline{\mathbf{5}}$ & $\square$ \\
$\bar{X}+\bar{Q}_{1}+Q_{2}$ & $\overline{\mathbf{1 0}}+\overline{\mathbf{5}}+\mathbf{5}$ & $\bar{\square}$ \\
\hline
\end{tabular}

Table 22. Couplings $T_{1} \bar{Q}_{1} \bar{Q}_{2}+T_{2} X Q_{2}$.

\begin{tabular}{|l|c|c|}
\hline & $\mathrm{SU}(5)_{\mathrm{GUT}}$ & $\mathrm{SU}(\mathrm{N})$ \\
\hline$X+S$ & $\mathbf{1 0}+\mathbf{1}$ & $\square$ \\
$\bar{X}+\bar{S}$ & $\overline{\mathbf{1 0}}+\mathbf{1}$ & $\bar{\square}$ \\
$A$ & $\mathbf{1}$ & $\mathrm{Ad}$. \\
\hline
\end{tabular}

Table 23. Couplings $T_{1} \bar{X} S+T_{2} \bar{X} A S$.

\begin{tabular}{|c|c|c|}
\hline & $\mathrm{SU}(5)_{\mathrm{GUT}}$ & $\mathrm{SU}(\mathrm{N})$ \\
\hline$X+S$ & $\mathbf{1 0}+\mathbf{1}$ & $\square$ \\
$\bar{X}+\bar{S}$ & $\overline{\mathbf{1 0}}+\mathbf{1}$ & $\bar{\square}$ \\
$B+\bar{B}$ & $\mathbf{1}$ & $(\mathrm{\theta}+\overline{\mathrm{G}})$ or $(\square+\bar{\square})$ \\
\hline
\end{tabular}

Table 24. Couplings $T_{1} \bar{X} S+T_{2} \bar{X} B \bar{S}$. 


\begin{tabular}{|c|c|c|}
\hline & $\mathrm{SU}(5)_{\mathrm{GUT}}$ & $\mathrm{SU}(\mathrm{N})$ \\
\hline$X+Q$ & $\mathbf{1 0}+\mathbf{5}$ & $\square$ \\
$\bar{X}+\bar{Q}$ & $\overline{\mathbf{1 0}}+\overline{\mathbf{5}}$ & $\bar{\square}$ \\
$B+\bar{B}$ & $\mathbf{1}$ & $\theta+\bar{G}$ \\
\hline
\end{tabular}

Table 25. Couplings $T_{1} X \bar{B} Q+T_{2} \bar{Q} B \bar{Q}$.

\begin{tabular}{|c|c|c|}
\hline & $\mathrm{SU}(5)_{\mathrm{GUT}}$ & $\mathrm{SU}(\mathrm{N})$ \\
\hline$X+\bar{Q}$ & $\mathbf{1 0}+\overline{\mathbf{5}}$ & $\square$ \\
$\bar{X}+Q$ & $\overline{\mathbf{1 0}}+\mathbf{5}$ & $\bar{\square}$ \\
$B+\bar{B}$ & $\mathbf{1}$ & $\boxminus+\bar{G}$ \\
\hline
\end{tabular}

Table 26. Couplings $T_{1} X Q+T_{2} \bar{Q} B \bar{Q}$.

\begin{tabular}{|c|c|c|}
\hline & $\mathrm{SU}(5)_{\mathrm{GUT}}$ & $\mathrm{SU}(\mathrm{N})$ \\
\hline$X+\bar{Q}$ & $\mathbf{1 0}+\overline{\mathbf{5}}$ & $\square$ \\
$\bar{X}+Q$ & $\overline{\mathbf{1 0}}+\mathbf{5}$ & $\bar{\square}$ \\
$A$ & $\mathbf{1}$ & $\mathrm{Ad}$. \\
\hline
\end{tabular}

Table 27. Couplings $T_{1} X Q+T_{2} X A Q$.

\begin{tabular}{|l|c|c|}
\hline & $\mathrm{SU}(5)_{\mathrm{GUT}}$ & $\mathrm{SO}(\mathrm{N})$ \\
\hline$X+\bar{X}+S$ & $\mathbf{1 0}+\overline{\mathbf{1 0}}+\mathbf{1}$ & $\square$ \\
$A$ & $\mathbf{1}$ & 日or $\square$ \\
\hline
\end{tabular}

Table 28. Couplings $T_{1} \bar{X} S+T_{2} \bar{X} A S$.

\begin{tabular}{|c|c|c|}
\hline & $\mathrm{SU}(5)_{\mathrm{GUT}}$ & $\mathrm{Sp}(2 \mathrm{~N})$ \\
\hline$X+\bar{X}+Q+\bar{Q}$ & $\mathbf{1 0}+\overline{\mathbf{1 0}}+\mathbf{5}+\overline{\mathbf{5}}$ & $\square$ \\
\hline
\end{tabular}

Table 29. Couplings $T_{1} \bar{Q} \bar{Q}+T_{2} X Q$. 


\section{References}

[1] C.D. Froggatt and H.B. Nielsen, Hierarchy of Quark Masses, Cabibbo Angles and CP-violation, Nucl. Phys. B 147 (1979) 277 [SPIRES].

[2] M. Leurer, Y. Nir and N. Seiberg, Mass matrix models, Nucl. Phys. B 398 (1993) 319 [hep-ph/9212278] [SPIRES].

[3] M. Leurer, Y. Nir and N. Seiberg, Mass matrix models: The Sequel, Nucl. Phys. B 420 (1994) 468 [hep-ph/9310320] [SPIRES].

[4] Y. Grossman and Y. Nir, Lepton mass matrix models, Nucl. Phys. B 448 (1995) 30 [hep-ph/9502418] [SPIRES].

[5] H. Georgi, A.E. Nelson and A. Manohar, On the proposition that all fermions are created equal, Phys. Lett. B 126 (1983) 169 [SPIRES].

[6] A.E. Nelson and M.J. Strassler, Suppressing flavor anarchy, JHEP 09 (2000) 030 [hep-ph/0006251] [SPIRES].

[7] L. Randall and R. Sundrum, A large mass hierarchy from a small extra dimension, Phys. Rev. Lett. 83 (1999) 3370 [hep-ph/9905221] [SPIRES].

[8] T. Gherghetta and A. Pomarol, Bulk fields and supersymmetry in a slice of AdS, Nucl. Phys. B 586 (2000) 141 [hep-ph/0003129] [SPIRES].

[9] S.J. Huber and Q. Shafi, Fermion Masses, Mixings and Proton Decay in a Randall-Sundrum Model, Phys. Lett. B 498 (2001) 256 [hep-ph/0010195] [SPIRES].

[10] J.M. Maldacena, The large-N limit of superconformal field theories and supergravity, Adv. Theor. Math. Phys. 2 (1998) 231 [Int. J. Theor. Phys. 38 (1999) 1113] [hep-th/9711200] [SPIRES].

[11] S.S. Gubser, I.R. Klebanov and A.M. Polyakov, Gauge theory correlators from non-critical string theory, Phys. Lett. B 428 (1998) 105 [hep-th/9802109] [SPIRES].

[12] E. Witten, Anti-de Sitter space and holography, Adv. Theor. Math. Phys. 2 (1998) 253 [hep-th/9802150] [SPIRES].

[13] N. Arkani-Hamed, M. Porrati and L. Randall, Holography and phenomenology, JHEP 08 (2001) 017 [hep-th/0012148] [SPIRES].

[14] R. Rattazzi and A. Zaffaroni, Comments on the holographic picture of the Randall-Sundrum model, JHEP 04 (2001) 021 [hep-th/0012248] [SPIRES].

[15] K.A. Intriligator and B. Wecht, The exact superconformal R-symmetry maximizes a, Nucl. Phys. B 667 (2003) 183 [hep-th/0304128] [SPIRES].

[16] Particle Data Group collaboration, C. Amsler et al., Review of particle physics, Phys. Lett. B 667 (2008) 1 [SPIRES].

[17] M.C. Gonzalez-Garcia, Neutrino Physics, Nucl. Phys. A 827 (2009) 5c-14c [arXiv:0901.2505] [SPIRES].

[18] S. Antusch and M. Spinrath, Quark and lepton masses at the GUT scale including SUSY threshold corrections, Phys. Rev. D 78 (2008) 075020 [arXiv:0804.0717] [SPIRES].

[19] J.L. Diaz-Cruz, H. Murayama and A. Pierce, Can supersymmetric loops correct the fermion mass relations in SU(5)?, Phys. Rev. D 65 (2002) 075011 [hep-ph/0012275] [SPIRES]. 
[20] T. Ibrahim and P. Nath, SUSY QCD and SUSY electroweak loop corrections to b, $t$ and $\tau$ masses including the effects of CP phases, Phys. Rev. D 67 (2003) 095003 [Erratum ibid. D 68 (2003) 019901] [hep-ph/0301110] [SPIRES].

[21] J. Ferrandis and N. Haba, Supersymmetry breaking as the origin of flavor, Phys. Rev. D 70 (2004) 055003 [hep-ph/0404077] [SPIRES].

[22] J.L. Diaz-Cruz, M. Gomez-Bock, R. Noriega-Papaqui and A. Rosado, A Numerical Analysis of the Supersymmetric Flavor Problem and Radiative Fermion Masses, Rev. Mex. Fis. 55 (2009) 270 [hep-ph/0512168] [SPIRES].

[23] G. Ross and M. Serna, Unification and Fermion Mass Structure, Phys. Lett. B 664 (2008) 97 [arXiv: 0704.1248] [SPIRES].

[24] C.M. Maekawa and M.C. Rodriguez, Radiative Mechanism to Light Fermion Masses in the MSSM, JHEP 01 (2008) 072 [arXiv:0710.4943] [SPIRES].

[25] T. Enkhbat, SU(5) unification for Yukawas through SUSY threshold effects, arXiv:0909.5597 [SPIRES].

[26] M. Dine and W. Fischler, A Phenomenological Model of Particle Physics Based on Supersymmetry, Phys. Lett. B 110 (1982) 227 [SPIRES].

[27] M. Dine and W. Fischler, A Supersymmetric GUT, Nucl. Phys. B 204 (1982) 346 [SPIRES].

[28] L. Álvarez-Gaumé, M. Claudson and M.B. Wise, Low-Energy Supersymmetry, Nucl. Phys. B 207 (1982) 96 [SPIRES].

[29] S. Dimopoulos and S. Raby, Geometric Hierarchy, Nucl. Phys. B 219 (1983) 479 [SPIRES].

[30] M. Dine, A.E. Nelson and Y. Shirman, Low-energy dynamical supersymmetry breaking simplified, Phys. Rev. D 51 (1995) 1362 [hep-ph/9408384] [SPIRES].

[31] M. Dine, A.E. Nelson, Y. Nir and Y. Shirman, New tools for low-energy dynamical supersymmetry breaking, Phys. Rev. D 53 (1996) 2658 [hep-ph/9507378] [SPIRES].

[32] A.E. Nelson and M.J. Strassler, Exact results for supersymmetric renormalization and the supersymmetric flavor problem, JHEP 07 (2002) 021 [hep-ph/0104051] [SPIRES].

[33] T. Kobayashi and H. Terao, Sfermion masses in Nelson-Strassler type of models: SUSY standard models coupled with SCFTs, Phys. Rev. D 64 (2001) 075003 [hep-ph/0103028] [SPIRES].

[34] R. Kitano and Y. Nomura, Supersymmetry with small mu: Connections between naturalness, dark matter and (possibly) flavor, hep-ph/0606134 [SPIRES].

[35] Y. Nomura, M. Papucci and D. Stolarski, Flavorful Supersymmetry, Phys. Rev. D 77 (2008) 075006 [arXiv:0712.2074] [SPIRES].

[36] Y. Nomura and D. Stolarski, Naturally Flavorful Supersymmetry at the LHC, Phys. Rev. D 78 (2008) 095011 [arXiv:0808.1380] [SPIRES].

[37] N. Arkani-Hamed and H. Murayama, Holomorphy, rescaling anomalies and exact $\beta$-functions in supersymmetric gauge theories, JHEP 06 (2000) 030 [hep-th/9707133] [SPIRES].

[38] N. Seiberg, Electric-magnetic duality in supersymmetric nonAbelian gauge theories, Nucl. Phys. B 435 (1995) 129 [hep-th/9411149] [SPIRES].

[39] G. Mack, All Unitary Ray Representations of the Conformal Group $\operatorname{SU}(2,2)$ with Positive Energy, Commun. Math. Phys. 55 (1977) 1 [SPIRES]. 
[40] M. Flato and C. Fronsdal, Representations of conformal supersymmetry, Lett. Math. Phys. 8 (1984) 159 [SPIRES].

[41] V.K. Dobrev and V.B. Petkova, All Positive Energy Unitary Irreducible Representations of Extended Conformal Supersymmetry, Phys. Lett. B 162 (1985) 127 [SPIRES].

[42] E. Barnes, K.A. Intriligator, B. Wecht and J. Wright, Evidence for the strongest version of the $4 D$ a-theorem, via a-maximization along $R G$ flows, Nucl. Phys. B 702 (2004) 131 [hep-th/0408156] [SPIRES].

[43] D. Anselmi, J. Erlich, D.Z. Freedman and A.A. Johansen, Positivity constraints on anomalies in supersymmetric gauge theories, Phys. Rev. D 57 (1998) 7570 [hep-th/9711035] [SPIRES].

[44] D. Kutasov, A. Parnachev and D.A. Sahakyan, Central charges and U(1)R symmetries in $N=1$ super Yang-Mills, JHEP 11 (2003) 013 [hep-th/0308071] [SPIRES].

[45] K.A. Intriligator and B. Wecht, $R G$ fixed points and flows in $S Q C D$ with adjoints, Nucl. Phys. B 677 (2004) 223 [hep-th/0309201] [SPIRES].

[46] D. Kutasov, New results on the 'a-theorem' in four dimensional supersymmetric field theory, hep-th/0312098 [SPIRES].

[47] C. Csáki, P. Meade and J. Terning, A mixed phase of SUSY gauge theories from a-maximization, JHEP 04 (2004) 040 [hep-th/0403062] [SPIRES].

[48] E. Barnes, K.A. Intriligator, B. Wecht and J. Wright, $N=1 R G$ flows, product groups and a-maximization, Nucl. Phys. B 716 (2005) 33 [hep-th/0502049] [SPIRES].

[49] K.A. Intriligator, IR free or interacting? A proposed diagnostic, Nucl. Phys. B 730 (2005) 239 [hep-th/0509085] [SPIRES].

[50] T. Kawano, Y. Ookouchi, Y. Tachikawa and F. Yagi, Pouliot type duality via a-maximization, Nucl. Phys. B 735 (2006) 1 [hep-th/0509230] [SPIRES].

[51] T. Kawano and F. Yagi, Supersymmetric $N=1$ Spin(10) Gauge Theory with Two Spinors via a-Maximization, Nucl. Phys. B 786 (2007) 135 [arXiv:0705.4022] [SPIRES].

[52] A.D. Shapere and Y. Tachikawa, A counterexample to the "a-theorem', JHEP 12 (2008) 020 [arXiv:0809.3238] [SPIRES].

[53] D. Poland, The Phase Structure of Supersymmetric $\mathrm{Sp}\left(2 \mathrm{~N}_{\mathrm{c}}\right)$ Gauge Theories with an Adjoint, JHEP 11 (2009) 049 [arXiv:0908.2131] [SPIRES].

[54] M.A. Shifman and A.I. Vainshtein, Solution of the Anomaly Puzzle in SUSY Gauge Theories and the Wilson Operator Expansion, Nucl. Phys. B 277 (1986) 456 [SPIRES].

[55] V.A. Novikov, M.A. Shifman, A.I. Vainshtein and V.I. Zakharov, Exact Gell-Mann-Low Function of Supersymmetric Yang-Mills Theories from Instanton Calculus, Nucl. Phys. B 229 (1983) 381 [SPIRES].

[56] V.A. Novikov, M.A. Shifman, A.I. Vainshtein and V.I. Zakharov, Supersymmetric instanton calculus: Gauge theories with matter, Nucl. Phys. B 260 (1985) 157 [SPIRES].

[57] M.A. Shifman, A.I. Vainshtein and V.I. Zakharov, Exact Gell-Mann-Low function in supersymmetric electrodynamics, Phys. Lett. B 166 (1986) 334 [SPIRES].

[58] L.J. Hall and Y. Nomura, A complete theory of grand unification in five dimensions, Phys. Rev. D 66 (2002) 075004 [hep-ph/0205067] [SPIRES].

[59] D. Kutasov, A. Schwimmer and N. Seiberg, Chiral Rings, Singularity Theory and Electric-Magnetic Duality, Nucl. Phys. B 459 (1996) 455 [hep-th/9510222] [SPIRES]. 
[60] D. Kutasov, A Comment on duality in $N=1$ supersymmetric nonAbelian gauge theories, Phys. Lett. B 351 (1995) 230 [hep-th/9503086] [SPIRES].

[61] K.A. Intriligator, New $R G$ fixed points and duality in supersymmetric $\mathrm{Sp}\left(\mathrm{N}_{\mathrm{c}}\right)$ and $\mathrm{SO}\left(\mathrm{N}_{\mathrm{c}}\right)$ gauge theories, Nucl. Phys. B 448 (1995) 187 [hep-th/9505051] [SPIRES].

[62] K.A. Intriligator and P. Pouliot, Exact superpotentials, quantum vacua and duality in supersymmetric $\mathrm{SP}\left(\mathrm{N}_{\mathrm{c}}\right)$ gauge theories, Phys. Lett. B 353 (1995) 471 [hep-th/9505006] [SPIRES].

[63] R. Kitano and G.D. Kribs, Tripletless unification in the conformal window, JHEP 03 (2005) 033 [hep-ph/0501047] [SPIRES].

[64] Y. Nomura, D. Poland and B. Tweedie, Holographic Grand Unification, JHEP 12 (2006) 002 [hep-ph/0605014] [SPIRES].

[65] R. Kitano, Dynamical GUT breaking and mu-term driven supersymmetry breaking, Phys. Rev. D 74 (2006) 115002 [hep-ph/0606129] [SPIRES].

[66] H. Osborn, $N=1$ superconformal symmetry in four-dimensional quantum field theory, Annals Phys. 272 (1999) 243 [hep-th/9808041] [SPIRES].

[67] S. Ferrara and B. Zumino, Transformation Properties Of The Supercurrent, Nucl. Phys. B 87 (1975) 207 [SPIRES].

[68] D. Anselmi, D.Z. Freedman, M.T. Grisaru and A.A. Johansen, Nonperturbative formulas for central functions of supersymmetric gauge theories, Nucl. Phys. B 526 (1998) 543 [hep-th/9708042] [SPIRES].

[69] K.A. Intriligator, N. Seiberg and D. Shih, Dynamical SUSY breaking in meta-stable vacua, JHEP 04 (2006) 021 [hep-th/0602239] [SPIRES].

[70] K.I. Izawa, F. Takahashi, T.T. Yanagida and K. Yonekura, Conformal Supersymmetry Breaking in Vector-like Gauge Theories, Phys. Rev. D 80 (2009) 085017 [arXiv:0905.1764] [SPIRES].

[71] T. Banks and A. Zaks, On the Phase Structure of Vector-Like Gauge Theories with Massless Fermions, Nucl. Phys. B 196 (1982) 189 [SPIRES].

[72] I.I. Kogan, M.A. Shifman and A.I. Vainshtein, Matching conditions and duality in $N=1$ SUSY gauge theories in the conformal window, Phys. Rev. D 53 (1996) 4526 [Erratum ibid. D 59 (1999) 109903] [hep-th/9507170] [SPIRES].

[73] M.A.A. van Leeuwen, A.M. Cohen and B. Lisser, LiE, A Package for Lie Group Computations, Computer Algebra Nederland, Amsterdam The Netherlands (1992), ISBN 90-74116-02-7.

[74] N. Craig, Simple Models of Superconformal Flavor, arXiv:1004.4218. 